Paul-Jean Cahen - Gabriel Picavet $₫$ -

Martine Picavet-L'Hermitte

\title{
Pointwise minimal extensions
}

Received: 28 August 2017 / Accepted: 11 February 2018 / Published online: 20 March 2018

(C) The Author(s) 2018

\begin{abstract}
We characterize pointwise minimal extensions of rings, introduced by Cahen et al. (Rocky Mt J Math 41:1081-1125, 2011), in the special context of domains. We show that pointwise minimal extensions are either integral or integrally closed. In the closed case, they are nothing but minimal extensions. Otherwise, there are four cases: either all minimal sub-extensions are of the same type (ramified, decomposed, or inert) or coexist as only ramified and inert minimal sub-extensions.
\end{abstract}

Mathematics Subject Classification $\quad 13 \mathrm{~B} 02 \cdot 13 \mathrm{~B} 21 \cdot 13 \mathrm{~B} 22 \cdot 13 \mathrm{~B} 30$

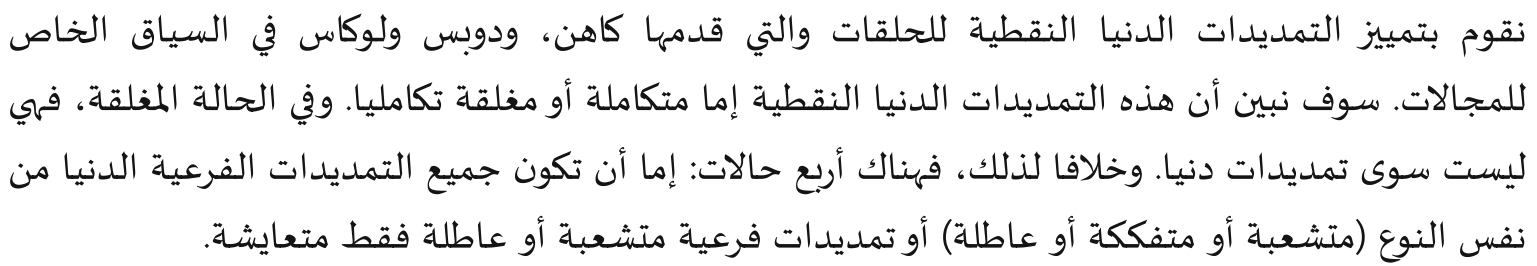

\section{Introduction}

All rings considered are commutative with identity and all rings morphisms and subrings are unital.

A ring extension $R \subset S$ is said to be a minimal extension if there is no ring properly between $R$ and $S$ (by ring between $R$ and $S$, we mean an $R$-subalgebra of $S$ ). Minimal extensions were introduced by Ferrand and Olivier [8] back in 1970, and from then on have often been considered, also in relation with chains of minimal extensions. One says that $R \subset S$ is a simple extension if $S=R[t]$ for some $t \in S$. Clearly, a minimal extension

P.-J. Cahen

12 Traverse du lavoir de grand mère, 13100 Aix en Provence, France

E-mail: paul-jean.cahen@univ-amu.fr

G. Picavet $(\varangle) \cdot$ M. Picavet-L'Hermitte

Mathématiques, 8 Rue du Forez, 63670 Le Cendre, France

E-mail: Gabriel.Picavet@math.univ-bpclermont.fr

M. Picavet-L'Hermitte

E-mail: picavet.mathu@orange.fr 
is simple, but the converse does not hold. Cahen et al. introduced pointwise minimal extensions, in the special context of domains (as a tool for the characterization of valuative domains [4]). An extension $R \subset S$ is said to be a pointwise minimal extension if every simple sub-extension $R \subset R[t]$ is minimal.

It is convenient to say that $x \in S$ is minimal (over $R$, but most often the context makes this precision clear) if $R \subset R[x]$ is a minimal extension, and that $x$ is trivial if $x \in R$. Thus, $R \subset S$ is pointwise minimal if and only if each $x \in S$ is either trivial or minimal. If $R \subset S$ is pointwise minimal, it is obvious that so is $R \subset T$ for each $T$ between $R$ and $S$, but this is not necessarily the case for $T \subset S$. We say that $R \subset S$ is a pointwise minimal pair if, for each $T$ between $R$ and $S, T \subset S$ is a pointwise minimal extension. We aim to characterize pointwise minimal extensions and pointwise minimal pairs, with no restriction to domains.

We first devote a section to useful recalls. Much is to be found in the seminal paper by Ferrand and Olivier [8] on minimal extensions and we briefly summarize all results that we later use. Obviously, if $R \subset S$ is minimal, then either $S$ is integral (hence finite) over $R$, or $R$ is integrally closed in $S$, and we simply say that $R \subset S$ is closed. We recall there are three types of minimal integral extensions and say that a minimal element $x \in S$ is ramified, decomposed, or inert according to the nature of the minimal extension $R \subset R[x]$. In the integral case, the conductor $M:=(R: S)$ is a maximal ideal of $R$. We thus also recall the basic properties of pairs of rings sharing an ideal; they are one of our main tools. We also recall the definitions of various chain properties (FCP, FIP, FMC, length of an extensions) that we later examine in relation to pointwise minimal extensions.

In Sect. 3, we deal with the general properties of pointwise minimal extensions. We show that, as for minimal extensions, a pointwise minimal extension is either integral or integrally closed. The closed case turns out to be trivial: an integrally closed pointwise minimal extension is in fact minimal. From then on, we thus focus on integral extensions and show that, as for integral minimal extensions, the conductor $M=(R: S)$ is a maximal ideal of $R$. We also characterize various chain properties of pointwise minimal extensions. We finally introduce co-pointwise minimal extensions as the extensions $R \subset S$, such that, for each nontrivial $x \in S$, $R[x] \subset S$ is minimal. We show they turn out to be the pointwise minimal extensions of length 2 .

By general properties of pairs of rings sharing an ideal, an extension $R \subset S$ sharing a maximal ideal $M$ of $R$, is pointwise minimal if and only if so is $R / M \subset S / M$. In Sect. 4, we thus focus on extensions over a field $k$. We first study the minimal sub-extensions in the general context of extensions over a field. We then turn to pointwise minimal extensions. We show that decomposed sub-extensions occur if and only if $S$ is not a local ring and never coexist with inert or ramified sub-extensions. Hence, there are four types of pointwise minimal extensions: decomposed, inert, ramified, where all sub-extensions are of the corresponding type, and composite, with both ramified and inert sub-extensions. We finally characterize each type of pointwise minimal extensions and pointwise minimal pairs over a field.

In Sect. 5 we turn back to general integral extensions. Applying the results of the previous section, we see there are four types of integral pointwise minimal extensions: ramified, decomposed, inert, and composite. We fully characterize each type of pointwise minimal extension as well as pointwise minimal pairs and copointwise minimal extensions. We examine various chain conditions and, for finite extensions, determine the length of pointwise minimal extensions of each type. In fact, we devote a whole subsection to the composite type for which saturated chains of different length always occur.

A last section is devoted to miscellaneous and examples. We give examples of pointwise minimal extensions over a field for each type, but the decomposed one (in this case, the characterization of pointwise minimal extensions is sufficiently explicit). We examine the transfer to Nagata rings and finally compare pointwise minimal extensions with quadratic extensions.

We set some terminology and notations. By local ring (elsewhere rather called a quasi-local ring), we just mean a ring with only one maximal ideal. If $k$ is a field and $S$ a $k$-algebra, the degree of $x \in S$ is the dimension $\operatorname{dim}_{k}(k[x])$.

The symbol $\subset$ always denotes proper containment. We denote by $\operatorname{char}(k)$ the characteristic of a field $k$. When we say that $\operatorname{char}(k)=p$, we mean that $p$ is a prime number. We denote by $\mathbb{F}_{2}$ the field with two elements and by $[L: k]$ the degree of a field extension $k \subset L$. If $A$ is a ring or an ideal of a ring and $n$ a positive integer, we set $A^{[n]}:=\left\{x^{n} \mid x \in A\right\}$ (we use this notation in contexts of fields, rings, and ideals).

\section{Useful results and recalls}

\subsection{Minimal extensions}

The dichotomy integral/integrally closed of minimal extensions is enlightened by [8, theorem 2.2]: 
Theorem 2.1 Let $R \subset S$ be a minimal extension.

(1) There exist a maximal ideal $M$ of $R$ (the crucial ideal of the extension) such that, for each prime $P \neq M$, $R_{P}=S_{P}$

(2) Either $R \subset S$ is finite and $M S=M$, or $R \subset S$ is closed and $M S=S$.

Ferrand and Olivier gave as trivial the fact that if $R \subset S$ is a minimal extension, then $R_{T} \subseteq S_{T}$ is minimal or trivial for every multiplicative subset $T$ of $S$ [8, Lemme 1.3]. The crucial ideal is then the only prime ideal such that $R_{P} \subset S_{P}$ is minimal. By globalization, one derives easily the following which allows to reduce to the local case [7, Proposition 2.1].

Corollary 2.2 An extension $R \subset S$ is minimal if and only if there is a maximal ideal $M$ of $R$ such that $R_{P}=S_{P}$ for each prime $P \neq M$ and $R_{M} \subset S_{M}$ is minimal.

In the integral case, the fact that $M S=M$ leads to another corollary [8, Proposition 4.1].

Corollary 2.3 An integral extension $R \subset S$ is minimal if and only if there is a maximal ideal $M$ of $R$ such that $M S=M$ and $R / M \subset S / M$ is minimal.

Minimal extensions over a field As $R / M$ is a field, one is led to consider extensions over a field. This is what Ferrand and Olivier did first. We quote them in their own terms (yet, that was in French!) [8, Lemma 1.2]:

Lemma 2.4 Let $k$ be a field and $f: k \rightarrow A$ be an injective morphism. Then, $f$ is minimal if and only if $f$ is of one of the three following types:

(1) A is a field and $k \rightarrow A$ is minimal.

(2) $f$ is the diagonal morphism $k \rightarrow k \times k$.

(3) $f$ is the canonical morphism $k \rightarrow D_{k}(k):=k[X] /\left(X^{2}\right)$.

In particular, A is a finite k-algebra.

It is trivial that if $k \subset A$ is minimal, then each $x \in A$ is algebraic over $k$, as otherwise $k \subset k\left[x^{2}\right] \subset k[x]$. If $R \subset S$ is a minimal integral extension with crucial maximal ideal $M$ and $k=R / M$, then $k \subset S / M$ is accordingly of one of these three types. It is customary to give them a name, as for instance in [5, Theorem 2.2], and first in [13, Definition 2.8]. (In fact, this terminology was suggested by Pierre Samuel for Martine Picavet-L'Hermitte' thesis back in 1986.)

(a) Inert: $S / M$ is a field and $k \subset S / M$ is a minimal field extension.

(b) Decomposed: $S / M \cong k \times k$.

(c) Ramified: $S / M \cong k[X] /\left(X^{2}\right)$.

Radicial extensions A minimal field extension $k \subset L$ is either separable or purely inseparable. In the first case, it can be of any degree $n \geq 2$ (for instance, the celebrated Eisenstein criterion provides irreducible polynomial of any degree over $\mathbb{Q}$ ). If the extension is purely inseparable, then char $(k)=p, L^{[p]} \subseteq k$, (that is, $x^{p} \in k$ for each $x \in L$ ), and $[L: k]=p$ [1, Proposition 1, A V.23].

More generally, we say that an extension $k \subset A$, where $k$ is a field of prime characteristic $p$ and $A$ a ring, is radicial of height one if $A^{[p]} \subseteq k$. This fits with more general definitions ${ }^{1}$ and, as we shall see, often occurs with pointwise minimal extensions. If $A^{[p]} \subseteq k$ then $k$ is assumed to be of prime characteristic $p$.

Closed extensions We finally summarize Ferrand and Olivier results on minimal closed extensions [8, Lemma 3.2 and Proposition 3.3]

Proposition 2.5 Let $R \subset S$ be a minimal closed extension. Then the conductor $P:=(R: S)$ is a prime ideal of both $R$ and $S, P$ is contained in the crucial maximal ideal $M$, and $(R / P)_{M}$ is a rank-one valuation domain.

\subsection{Chain conditions}

Given a ring extension $R \subset S$, a chain is a linearly ordered set of rings ( $R$-subalgebras of $S$ ) between $R$ and $S$. A (finite) chain from $R$ to $S, R=R_{0} \subset R_{1} \subset \cdots \subset R_{n-1} \subset R_{n}=S$, is said to be of length $n$, and it is

\footnotetext{
${ }^{1}$ A purely inseparable field extension $K \subset L$ is called radicial (radiciel in French) of height one if $L^{[p]} \subseteq K$, of height two, if not of height one, but $L^{\left[p^{2}\right]} \subseteq K$, etc. A ring morphism $R \rightarrow S$ is called radicial if $\operatorname{Spec}\left(R^{\prime} \otimes_{R} S\right) \rightarrow \operatorname{Spec}\left(R^{\prime}\right)$ is injective for any base change $R \rightarrow R^{\prime}$. This is the case if and only if $\operatorname{Spec}(S) \rightarrow \operatorname{Spec}(R)$ is injective and all residual extensions are radicial [9, Proposition 3.7.1]. In fact, a radicial field extension is nothing but a radicial ring extension.
} 
said to be saturated if, for each $i$, one cannot add any ring between $R_{i}$ and $R_{i+1}$ (that is, each step $R_{i} \subset R_{i+1}$ is a minimal extension).

We then recall the following definitions.

Definition 2.6 Let $R \subset S$ be a ring extension.

(1) The extension is said to have FMC (for a finite maximal chain property) if there is a finite saturated chain from $R$ to $S$.

(2) The extension $R \subseteq S$ is said to have $F C P$ (for the finite chain property) if each chain from $R$ to $S$ is finite.

(3) The extension $R \subseteq S$ is said to have FIP (for the finitely many intermediate algebras property) if the set of rings between $R$ and $S$ is finite.

(4) The length of the extension, denoted by $\ell[R, S]$, is the supremum of the lengths of chains from $R$ to $S$.

It is obvious that FIP implies FCP and that FCP implies FMC. ${ }^{2}$ A minimal extension is nothing but an extension of length one.

\subsection{Pairs of rings sharing an ideal}

One says that $R \subset S$ share an ideal $I$, when $I$ is an ideal of both $R$ and $S$. Properties of pairs of rings sharing an ideal [2] are used all along this paper. This situation can equally be seen as a pullback, represented by the following diagram (also called a cartesian square).

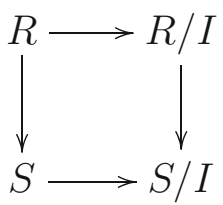

If $\varphi$ is the canonical map $S \rightarrow S / I$, then $R=\varphi^{-1}(R / I)$. The following is absolutely elementary, but fundamental:

Lemma 2.7 Let $R \subset S$ be a pair of rings sharing an ideal $I$. Then the set of rings between $R$ and $S$ is in one-to-one order preserving correspondence with the set of rings between $R / I$ and $S / I$.

Almost all properties of the extension $R \subset S$ that we wish to consider are thus perfectly reflected by the extension $R / I \subset S / I$.

Proposition 2.8 Let $R \subset S$ be a pair of rings sharing an ideal I. Then, $R \subset S$ is a minimal extension, or has $F C P, F M C$, FIP, or has length $n$, if and only if the same property holds for the extension $R / I \subset S / I$.

Conductor If $R \subset S$ share an ideal $I$, then $I S=I$, a fortiori $I$ is contained in the conductor $(R: S)$. Hence, $(R: S)$ is the largest ideal shared by $R$ and $S$. We recall the following easy result.

Proposition 2.9 Let $R \subset S$ be a pair of rings sharing an ideal $I$. Then, $R_{P}=S_{P}$ for each prime $P$ of $R$ that does not contain $I$, a fortiori that does not contain $(R: S)$.

If the conductor is trivial, that is, $(R: S)=(0)$, or more generally contained in the nilradical of $R$, then all primes contain $(R: S)$. But if $R$ is a domain and $R \subset S$ shares a nontrivial ideal $I$, it follows from the previous proposition that $S$ is an overring of $R$ (that is, a domain with the same quotient field as $R$ ).

Crucial ideal and critical ideal We give a general definition of the crucial ideal. We also define the critical ideal as introduced in [3].

Definition 2.10 Let $R \subset S$ be an extension.

(1) A (necessarily maximal) prime ideal $M$ is said to be crucial if $R_{P}=S_{P}$ for each prime $P \neq M$.

(2) A (necessarily prime) ideal $P$ is said to be critical if, for each nontrivial $t \in S, \sqrt{(R: t)}=P$.

By definition, the crucial and the critical ideal (if any) are both unique. If there is a crucial ideal $M$, it is clearly maximal (the set of primes such that $R_{P} \neq S_{P}$ is the support of the $R$-module $S / R$ ). If there is a critical ideal $P$, it is a radical ideal by definition; in fact, it is prime [3, Lemma 2.11], and may be not maximal (let $R=\mathbb{Z}$ and $S$ be the ring of integers of a number field. For each $t \in S \backslash R,(R: t)=(0))$. But for a maximal ideal, both notions coincide:

\footnotetext{
${ }^{2}$ It is less trivial that FCP implies that the length of the extension is finite and there is a (necessarily saturated) chain of length $\ell[R, S][6$, Theorem 4.11].
} 
Proposition 2.11 Let $R \subset S$ be an extension and $M$ be a maximal ideal of $R$. Then, $M$ is crucial if and only if it is critical.

Proof Let $t \in S$ be nontrivial and $P$ be a prime ideal. Clearly, $R_{P}[t]=R_{P}$, if and only if $(R: t)$ is not contained in $P$. Thus, $\sqrt{(R: t)}=M$ for each nontrivial $t$, if and only if $R_{P}=S_{P}$ for each $P \neq M$.

Theorem 2.1 shows that a minimal extension has a crucial, thus also critical, maximal ideal $M$ (see also [3, Proposition 2.14, case (2)]).

\subsection{The canonical decomposition of an integral extension}

We recall the basic definitions and results about the canonical decomposition of an integral extension [11,14]. We link these notions with pointwise minimal extensions in Sect. 4; otherwise, we do not use them, and the reader may skip this subsection.

Definition 2.12 Let $R \subseteq S$ be an integral extension.

(1) $R \subseteq S$ is said to be infra-integral if all its residual extensions are isomorphisms.

(2) $R \subseteq S$ is said to be subintegral if it is infra-integral and the spectral map $\operatorname{Spec}(S) \rightarrow \operatorname{Spec}(R)$ is bijective.

(3) $R \subseteq S$ is said to be seminormal if $b \in S, b^{2} \in R$, and $b^{3} \in R$ implies $b \in R$.

(4) $R \subseteq S$ is said to be $t$-closed if $b \in S, r \in R, b^{2}-r b \in R$, and $b^{3}-r b^{2} \in R$ implies $b \in R$.

By definition, subintegral implies infra-integral, and obviously, t-closed implies seminormal. In case $R \subset S$ share an ideal $I$, all these properties are reflected in $R / I \subset S / I$, as others (Proposition 2.8).

Proposition 2.13 Let $R \subset S$ be a pair of rings sharing an ideal I. Then $R \subset S$ is infra-integral, subintegral, seminormal, or $t$-closed if and only if the same property holds for the extension $R / I \subset S / I$.

We often focus on extensions $k \subset S$ over a field $k$. Clearly, $k \subset S$ is infra-integral if and only if, for every maximal ideal $M$ of $S, S / M \cong k$. The extension is subintegral if moreover $S$ is a (zero-dimensional) local ring. It is seminormal if and only if $S$ is a reduced ring. ${ }^{3}$ Finally, it is not too difficult to see that $k \subset S$ is t-closed if and only if $S$ is a field [10, Lemme 3.10]. In particular, we thus have the following for minimal extensions.

Proposition 2.14 Let $k$ be a field and $k \subset S$ be a minimal extension. Then, $k \subset S$ has one of three mutually exclusive properties:

(1) $k \subset S$ is $t$-closed in the inert case ( $S$ is a field),

(2) $k \subset S$ is seminormal and infra-integral in the decomposed case $(S \cong k \times k)$,

(3) $k \subset S$ is subintegral in the ramified case $\left(S \cong k[X] /\left(X^{2}\right)\right)$.

Given a family $\left\{B_{i}\right\}_{i \in I}$ of elements of rings between $R$ and $S$ such that, for each $i \in I, B_{i} \subseteq S$ is t-closed (resp. seminormal), it is not too difficult to see that $\bigcap_{i \in I} B_{i} \subseteq S$ is t-closed (resp. seminormal). This leads to two more definitions:

Definition 2.15 Let $R \subseteq S$ be an integral extension.

(1) The t-closure ${ }_{S}^{t} R$ (of $R$ in $S$ ) is the smallest ring $B$ between $R$ and $S$ such that $B \subseteq S$ is t-closed.

(2) The seminormalization ${ }_{S}^{+} R$ (of $R$ in $S$ ) is the smallest $\operatorname{ring} B$ between $R$ and $S$ such that $B \subseteq S$ is seminormal.

In fact, the t-closure ${ }_{S}^{t} R$ (resp. the seminormalization ${ }_{S}^{+} R$ ) is also the largest ring $B$ between $R$ and $S$ such that $B \subseteq S$ is infra-integral (resp. subintegral) [11, Theorem 2.1] and [14, Lemma 2.2].

As t-closed implies seminormal, one has the following containments

$$
R \subseteq{ }_{S}^{+} R \subseteq{ }_{S}^{t} R \subseteq S
$$

This is known as the canonical decomposition of the extension.

\footnotetext{
${ }^{3}$ In general, if $R \subset S$ is seminormal, then $(R: S)$ is a radical ideal in both $R$ and $S$ (the proof goes back to Traverso [15, Lemma 1.3]) .
} 


\section{General properties on pointwise minimal extensions}

\subsection{From minimal to pointwise minimal extensions}

We show that pointwise minimal extensions behave pretty much like minimal extensions. But we first settle trivialities, generalizing [4, Propositions 4.2 and 4.7] with the added consideration of pointwise minimal pairs.

Proposition 3.1 Let $R \subset S$ be an extension. Then,

(1) If $R \subset S$ is a pointwise minimal extension (resp. pair), then every sub-extension $R \subset T$ is pointwise minimal (resp. every sub-extension $T \subset T^{\prime}$ is a pointwise minimal pair).

(2) If $R$ and $S$ share an ideal $I$, then $R \subset S$ is a pointwise minimal extension (resp. pair) if and only if $R / I \subset S / I$ is a pointwise minimal extension (resp. pair).

Crucial ideal and dichotomy The dichotomy integral/integrally closed (Theorem 2.1) extends (almost) perfectly to pointwise minimal extensions. However, unlike minimal extensions, an integral pointwise minimal extension need not be finite.

Theorem 3.2 Let $R \subset S$ be a pointwise minimal extension.

(1) There exists a maximal ideal $M$ of $R$ (the crucial ideal of the extension) such that, for each prime $P \neq M$, $R_{P}=S_{P}$

(2) Either $R \subset S$ is integral and $M S=M$, or $R \subset S$ is closed and $M S=S$.

Proof (1) Using Proposition 2.11, we show there is a maximal critical ideal. The argument is the same as in [3, Proposition 2.14, case (3)]. For each nontrivial $t \in S, \sqrt{(R: t)}$ is a maximal ideal (the crucial ideal of the minimal extension $R \subset R[t])$. Given two nontrivial elements $t$ and $s$, we show that $\sqrt{(R: t)}=\sqrt{(R: s)}$. Let $r=s+t$. If $r \in R$, then $(R: t)=(R: s)$, and we are done. Otherwise, $\sqrt{(R: t)}, \sqrt{(R: s)}$, and $\sqrt{(R: r)}$ are maximal ideals. Each one contains the intersection of the other two; thus, they are equal to a same maximal ideal $M$. Hence, $M$ is the critical, then also crucial, ideal of the extension.

(2) We now show that $R \subset S$ is either integral or integrally closed. ${ }^{4}$ By way of contradiction, suppose there are both a closed sub-extension $R \subset R(t]$ and a finite one $R \subset R[s]$. By Theorem 2.1, $M R[t]=R[t]$ and $M R[s]=M$. Therefore,

$$
R[s] \subseteq R[t] R[s]=(M R[t]) R[s]=R[t](M R[s])=M R[t]=R[t] .
$$

We reach a contradiction. In the integral case, $M R[t]=M$ for each $t \in S$, a fortiori $M t \subseteq M$. Thus, $M S=M$. In the closed case, $t \in M R[t]$ for each $t \in S$, a fortiori $t \in M S$. Thus $M S=S$.

Generalizing [4, Theorem 4.5], we derive a corollary perfectly similar to Corollary 2.2.

Corollary 3.3 An extension $R \subset S$ is pointwise minimal if and only if there is a maximal ideal $M$ of $R$ such that $R_{P}=S_{P}$ for each prime $P \neq M$ and $R_{M} \subset S_{M}$ is pointwise minimal.

And in the integral case, the following is quite similar to Corollary 2.3.

Corollary 3.4 An integral extension $R \subset S$ is pointwise minimal if and only if there is a maximal ideal $M$ of $R$ such that $M S=M$ and $R / M \subset S / M$ is a pointwise minimal extension.

This allows to focus on extensions over a field, as in the next section.

Closed extensions The closed case turns out to be trivial.

Proposition 3.5 An integrally closed extension $R \subset S$ is pointwise minimal if and only if it is minimal.

Proof One implication is obvious. For the converse, we use an argument similar to the proof of Theorem 3.2. Suppose that $R \subset S$ is pointwise minimal with crucial ideal $M$. From Corollaries 2.2 and 3.3, we may as well assume that $R$ is local, with maximal ideal $M$. As the extension is closed, it follows by Proposition 2.5 that, for each nontrivial $t \in S, P_{t}:=(R: R[t])$ is a prime ideal of $R$ and $R / P_{t}$ is a rank-one valuation domain. Let $r, s \in S$ be two nontrivial elements. We show that $P_{t}=P_{s}$. Let $r=s+t$. If $r \in R$, then $R[t]=R[s]$ and we are done. Otherwise, $R \subset R[r]$ is minimal, and thus $P_{r}:=(R: R[r])$ is a prime ideal such that $R / P_{r}$ is a rank-one valuation domain. Each prime $P_{t}, P_{s}, P_{r}$ contains the product of the other two. As all three are properly contained in no other prime ideal than $M$, they are equal to a same prime $P$. Therefore, $P=(R: S)$. As $R / P$ is a rank-one valuation domain, $R / P \subset S / P$ is a minimal extension and, finally, so is $R \subset S$ by Proposition 2.8.

From then on, we shall only consider integral extensions.

${ }^{4}$ This follows from [4, Proposition 4.13] in the case of local domains. 


\subsection{Chain conditions}

We now deal with chain properties, first in a more general context than pointwise minimal extensions.

Proposition 3.6 Let $R \subset S$ be an integral extension sharing an ideal $M$ maximal in $R$. Then all the following properties are equivalent: $S$ is a finitely generated $R$-algebra, the extension $R \subset S$ is finite, $R \subset S$ has $F C P$, $R \subset S$ has $F M C, \ell[R, S]<\infty, \operatorname{dim}_{R / M}(S / M)<\infty$.

Proof By Proposition 2.8, all properties of $R \subset S$ are reflected in the residual extension $R / M \subset S / M$. Given a field $k$, an algebraic $k$-algebra $A$ is finitely generated if and only if $\operatorname{dim}_{k}(A)<\infty$. All other implications are obvious.

We finally deal with the FIP property.

Proposition 3.7 Let $R \subset S$ be a pointwise minimal extension with crucial ideal $M$, and $k:=R / M$. Then, $R \subset S$ has FIP if and only if either $R \subset S$ is minimal or $k$ is a finite field and the extension is finite.

Proof Suppose $R \subset S$ has FIP and is not minimal. We can thus assume that $R \subset S$ is an integral extension (Theorem 3.2 and Proposition 3.5). Then, $k \subset S / M$ has FIP and is not minimal. There exist two minimal elements $s, t$ in $S / M$ such that $k[s] \neq k[t]$. By way of contradiction, suppose that $k[s+a t]=k[s+b t]$ with $a \neq b$. Then $(a-b) t \in k[s+a t]$. As $(a-b)$ is a unit in $k, t$ belongs to $k[s+a t]$, so does then $s$. This leads to $k[s]=k[t]$ and we reach a contradiction. Therefore, if $a$ runs through $k$, all rings $k[s+a t]$ are distinct. Hence, $k$ must be a finite field. On the other hand, FIP implies FCP, and by Proposition 3.6, the extension is finite. Conversely, a minimal extension obviously has FIP, and if $k$ is a finite field and the extension is finite, then $S / M$ is a finite set.

\subsection{Co-pointwise minimal extensions}

We say that $R \subset S$ is a co-pointwise minimal extension if, for each nontrivial $x \in S, R[x] \subset S$ is minimal. A weaker condition is that $R[x] \subseteq S$ is minimal or trivial. In particular, a minimal extension is not co-pointwise minimal, but satisfies this condition. We first characterize the weaker condition.

Lemma 3.8 An extension $R \subset S$ is such that, for each nontrivial $x \in S, R[x] \subseteq S$ is minimal or trivial, if and only if $l[R, S] \leq 2$.

Proof Assume that $\ell[R, S] \leq 2$. Let $x \in S$ be a nontrivial element. Either $R[x]=S$, or the chain $R \subset$ $R[x] \subset S$ is saturated; in particular, $R[x] \subset S$ is minimal. Conversely, let $R \subset T \subset S$ be a chain of length 2 (if any). Let $x \in T$ be a nontrivial element. By hypothesis $R[x] \subset S$ is minimal. As $R[x] \subseteq T \subset S$, it follows that $T \subset S$ is minimal. By way of contradiction, suppose that $R \subset T$ is not minimal: there is $T^{\prime}$ with $R \subset T^{\prime} \subset T \subset S$. But then, $T^{\prime} \subset S$ is not minimal, contrary to the previous argument applied to $T^{\prime}$.

Proposition 3.9 Let $R \subset S$ be an extension. The following conditions are equivalent:

(1) $R \subset S$ is a co-pointwise minimal extension,

(2) $\ell[R, S]=2$ and, for each $x \in S \backslash R, R[x] \neq S$,

(3) $\ell[R, S]=2$ and $R \subset S$ is a pointwise minimal extension,

(4) $R \subset S$ is a pointwise minimal pair and $S=R[x, y]$ where $(x, y)$ is a minimal system of generators.

Proof By the previous lemma, (1) and (2) are equivalent. So are easily (2) and (3).

Assume (3). If $T$ is such that $R \subset T \subset S$, then $\ell[R, S]=2$ implies that $T \subset S$ is minimal, and thus trivially pointwise minimal. As $R \subset S$ itself is pointwise minimal, it follows that $R \subset S$ is a pointwise minimal pair. Now, take $x \in S \backslash R$ and $y \in S \backslash R[x]$. Then, $R \subset R[x] \subset R[x, y]=S$ and $(x, y)$ is a minimal system of generators.

Assume (4). We show that a nontrivial element $z \in S$ is such that $R[z] \subset S$ is minimal. As $(x, y)$ is a minimal system of generators, and $R \subset S$ is a pointwise minimal pair, $R \subset R[x] \subset R[x, y]=S$ and both $R \subset R[x]$ and $R[x] \subset R[x, y]=S$ are minimal extensions. We then consider two cases:

- If $R[z]=R[x]$, we are done.

- Otherwise, $x \notin R[z]$ and $z \notin R[x]$, as $R \subset S$ is pointwise minimal. Thus, $R[x] \subset R[x, z] \subseteq S$, so that $R[x, z]=S$, since $R[x] \subset S$ is minimal. Finally, $R[z] \subset R[x, z]=S$ is minimal, since $R \subset S$ is a pointwise minimal pair. 
Remark 3.10 One cannot replace pointwise minimal pair by pointwise minimal extension in condition (4) (see Example 6.1).

\section{Integral extension over a field}

In this section, we consider extensions over a field $k$. Note that decomposed and ramified elements are then of degree 2 , that a nontrivial idempotent $e$ is decomposed, and that a nilpotent element $x$ is ramified if and only if it is of degree 2 , that is, $x \neq 0$, and $x^{2}=0$.

\subsection{Minimal sub-extensions}

We consider an extension $k \subset S$, not necessarily pointwise minimal, and first discuss its minimal subextensions. Most often we restrict to integral extensions. In this case, every finite sub-extension is finite dimensional, and this shows trivially there are minimal sub-extensions. This is worth recording.

Proposition 4.1 Let $k \subset S$ be an integral extension over a field. Then every finite sub-extension, in particular every simple sub-extension, contains a minimal sub-extension.

Decomposed minimal sub-extensions We give a simple condition for the existence of decomposed minimal sub-extensions.

Lemma 4.2 Let $k \subset S$ be an integral extension over a field. There is a minimal decomposed sub-extension if and only if $S$ is not a local ring.

Proof Suppose there is a decomposed sub-extension $k \subset k[x]$. Then $k \mid x] \cong k \times k$ has two maximal ideals, and these ideals lift in $S$. Conversely, if $S$ is not a local ring, there are distinct maximal ideals $M$ and $N$ and thus also $x \in S$ such that $x \in M, x \notin N$. Then, $k \subset k[x]$ is finite and not local, thus $k[x]$ is artinian and a product of more than one local ring. Hence, $k[x]$ contains a nontrivial idempotent $e$ and $k \subset k[e]$ is minimal decomposed.

We next consider the case where there are both decomposed and either ramified or inert minimal subextensions (we do not need to assume that $k \subset S$ is an integral extension). Note that there is a decomposed (resp. ramified) minimal sub-extension if and only if there is a nontrivial idempotent (resp. a nilpotent element of degree 2).

Lemma 4.3 Let $k \subset S$ be an extension over a field.

(1) If $e \in S$ is a nontrivial idempotent and $x \in S$ a nilpotent element of degree 2, then $(e+x)$ is neither trivial nor minimal.

(2) If $e \in S$ is a nontrivial idempotent and $y \in S$ is inert, then ey is neither trivial nor minimal.

Proof (1) The relations $e^{2}=e, x^{2}=0$, bring $(e+x)^{2}=e+2 e x$, and $(e+x)^{3}=e+3 e x$. The difference $e x$ is in $k[e+x]$. Then, $e \in k[e+x]$ and, in turn, $x \in k[e+x]$. Hence, $e+x$ is not trivial. It is not minimal as $e$ is decomposed and $x$ is ramified.

(2) We claim that $1, e y,(e y)^{2}$ are linearly independent. Consider a relation $a+b e y+c(e y)^{2}=0$ with $a, b, c \in k$. Then, $e^{2}=e$ brings $x:=e y(b+c y) \in k$. Thus, either $x=0$ or $x$ is a unit in $k$. Suppose $x$ is a unit in $k$, a fortiori in $S$, then $e$ is a unit. We reach a contradiction, and thus $x=0$. As $k[y]$ is a field, $(b+c y)$ is either a unit or 0 . Suppose $(b+c y)$ is a unit, so is $y(b+c y)$. Then, $x=0$ implies $e=0$. We reach a contradiction, and thus $b+c y=0$. As $y \notin k$, we then have $b=c=0$. Our claim is proved. In particular, ey is not trivial. As ey is of degree at least 3, it is neither ramified nor decomposed. As ey is not a unit (as above for $x$ ), it is also not inert. So, ey is not minimal.

In case $k \subset S$ is pointwise minimal, it follows that decomposed minimal sub-extensions cannot coexist with other ones. But in general, all three types may coexist, as shown by the following example.

Example 4.4 Let $k \subset L$ be an algebraic field extension and $S$ be the $k$-algebra $S:=L \times L[X] /\left(X^{2}\right)$. By Proposition 4.1, $L$, a fortiori $S$, contains inert elements, and clearly $S$ contains also nontrivial idempotents and non-zero nilpotent elements of degree 2. 
Ramified and inert sub-extensions If $k \subset S$ is an integral extension, then $S$ is zero-dimensional. The nilradical $N$ of $S$ is also the Jacobson radical of $S$.

Proposition 4.5 Let $k \subset S$ be an extension over a field and $N$ be the nilradical of $S$. A minimal element $x \in S$ is ramified if and only if $x \in k+N$, otherwise inert or decomposed. In particular:

(1) if $S=k+N$, all minimal sub-extensions are ramified,

(2) no minimal sub-extension is ramified if and only if $S$ is reduced,

(3) all minimal sub-extensions are inert if and only if $S$ is a field,

(4) if ramified sub-extensions coexist with inert or decomposed ones, then $k \subset k+N \subset S$.

Proof If $x \in S$ is ramified, then $k[x] \cong k[X] /\left(X^{2}\right)$, and $x=a+n$ with $a \in k$ and $n$ nilpotent of degree 2 . In particular, $x \in k+N$. Conversely if $x \in k+N$ is minimal, then $x$ is not decomposed, because $k+N$ is a local ring (with maximal ideal $N$ ) and not inert, because there is no field $L$ such that $k \subset L \subseteq k+N$. Hence, $x$ is ramified. If in particular, $S=k+N$, then all minimal elements are ramified.

2) $S$ is reduced if and only if $N=(0)$, that, is each $x \in k+N$ is trivial. If this is the case, then no element is ramified. If not, there exist ramified elements by Proposition 4.1 .

3) If $S$ is a field, then $k[y]$ is a field for each $y \in S$, and all minimal sub-extensions are inert. Conversely, if all minimal sub-extensions are inert, then none are decomposed; thus, $S$ is a (zero-dimensional) local ring, and none are ramified and thus $S$ is reduced. Therefore, $S$ is a field.

4) Follows immediately from 1) and 2).

Remark 4.6 If $k \subset S$ is pointwise minimal, then all elements outside $k+N$ are minimal, and thus inert or decomposed. But if $k \subset S$ is not pointwise minimal, the elements outside $k+N$ may fail to be minimal. Hence, one can have $k \subset k+N \subset S$ and all minimal sub-extensions ramified (see Example 6.5).

In case there are both ramified and inert elements, we have the following.

Lemma 4.7 Let $k \subset S$ be an integral extension over a field. If $x \in S$ is nilpotent of degree 2 and $y \in S$ is inert, then $(x+y)$ is not trivial. If $(x+y)$ is minimal, then char $(k)=p$ and $y^{p} \in k$.

Proof Let $N$ be the nilradical of $S$. Clearly, $x \in k+N$ and $y \notin k+N$. Thus, $(x+y) \notin k+N$; in particular, $(x+y)$ is not trivial and, by Proposition 4.5, if it is minimal, it is either decomposed or inert. Suppose that $(x+y)$ is decomposed. Then, $(x+y)$ is of degree 2 and there is a nontrivial idempotent $e$ such that $e=a+b(x+y)$, with $a, b \in k, b \neq 0$. Then, $e-b x=a+b y$ is minimal (inert). By Lemma 4.3, we reach a contradiction as $e$ is a nontrivial idempotent and $(-b x)$ a nilpotent element of degree 2. So, if $(x+y)$ is minimal, it is inert. In that case, let $P$ be the minimal polynomial of $(x+y)$ and $P^{\prime}$ its derivative. Then, $P(x+y)=0$ and $x^{2}=0$ bring $P(y)+x P^{\prime}(y)=0$. As $\{1, x\}$ is clearly a basis of the $k[y]$-vector space $k[x, y]$, it follows that $P(y)=P^{\prime}(y)=0$. As $P$ is irreducible, $P$ must be the minimal polynomial of $y$, and $P^{\prime}(y)=0$ implies $P^{\prime}=0$. In particular, the field extension $k \subset k[y]$ is not separable. Thus, $\operatorname{char}(k)=p$, for some prime $p$. As $y$ is minimal, it follows that $y^{p} \in k$ (and also $(x+y)^{p}=0$ ).

Example 4.8 The extension $\mathbb{R} \subset S=\mathbb{C}[X] /\left(X^{2}\right)$ is finite (more precisely, $\operatorname{dim}_{\mathbb{R}}(S)=4$ ). Clearly, $i \in \mathbb{C}$ is inert, and the class $x$ of $X$ is nilpotent of degree 2 . As $\operatorname{char}(\mathbb{R})=0$, it follows that $(i+x)$ is not minimal. In fact, it is easy to see that $\mathbb{R}[i+x]=S$.

\subsection{Pointwise minimal extensions}

For pointwise minimal extensions, the results of the previous subsection become simpler.

The four types It follows from Lemma 4.3 that either all or no minimal sub-extensions are decomposed. Hence, there are four types of pointwise minimal extensions. We give them a name with a definition (for later use) in the more general context of integral extensions.

Definition 4.9 Let $R \subset S$ be a pointwise minimal integral extension. We say the extension is of the decomposed type, or simply decomposed (resp. inert, resp. ramified), if all minimal sub-extensions are decomposed (resp. inert, resp. ramified). We say it is composite if there are both ramified and inert sub-extensions.

From Lemma 4.2, and Proposition 4.5, we derive immediately the following.

Corollary 4.10 Let $k \subset S$ be a pointwise minimal extension over a field. 
(1) If $S$ is not a local ring, then $k \subset S$ is decomposed.

(2) If $S$ is a local ring with maximal ideal $N$, then all minimal sub-extensions are either ramified or inert. More precisely, the extension is inert if and only if $S$ is a field, ramified if and only if $S=k+N$, and composite if and only if $k \subset k+N \subset S$.

We shall see that all four cases may effectively occur.

Necessary conditions We derive more necessary conditions from the previous subsection.

Proposition 4.11 Let $k \subset S$ be a pointwise minimal extension over a field.

(1) If $k \subset S$ is decomposed, then $S$ is a reduced ring and each residue field of $S$ is isomorphic to $k$.

(2) If $S$ is a local ring with maximal ideal $N$, then $N^{[2]}=(0)$.

(3) If $S$ is composite, then $\operatorname{char}(k)=p$ and $S^{[p]} \subseteq k$.

Proof (1) If $k \subset S$ is decomposed, each nontrivial $x$ is such that $k[x] \cong k \times k$. Thus, $S$ is reduced. Let $M$ be a maximal ideal of $S$. For each $x \in S$, the class of $x$ modulo $M$ belongs to $k$ (trivially if $x \in k$, otherwise because $k[x] \cong k \times k$ ). Thus, $S / M \cong k$.

(2) In the local case, as $S$ is zero-dimensional, if $x \in N$, then $x$ is nilpotent. Thus, either $x=0$, or $x$ is minimal ramified. In any case, $x^{2}=0$.

(3) If $S$ is composite, then $S$ is local with maximal ideal $N$; it follows by Lemma 4.7 that char $(k)=p$ and every inert $y$ is such that $y^{p} \in k$. By Proposition 4.5, we are left to consider the elements in $k+N$. If $x=a+n$, with $a \in k, n \in N$, then $n^{2}=0$ and $x^{p}=a^{p}+n^{p}=a^{p} \in k$. Therefore, $S^{[p]} \subseteq k$ (that is, $k \subset S$ is radicial of height one).

It is immediate that $S$ is a reduced ring if and only if $k \subset S$ is seminormal and, by definition, that $k \subset S$ is infra-integral if and only if all the residue fields of $S$ are isomorphic to $k$ (Definition 2.12). We give below a characterization of seminormal infra-integral extensions but, before that, we recall that a ring is said to be Von Neumann regular if for each $x \in S$, there is $a \in S$ such that $x=x a x$ (the definition extends to noncommutative rings) and that $S$ is said to be Boolean if every element is an idempotent.

Proposition 4.12 Let $k \subset S$ be an integral extension over a field. The following assertions are equivalent:

(1) $S$ is reduced and each residue field of $S$ is isomorphic to $k$.

(2) Each finite sub-extension $k \subset T$ is such that $T \cong k^{n}$ for some $n \geq 2$.

Under these equivalent conditions, $S$ is Von Neumann regular. If moreover $k=\mathbb{F}_{2}$, then $S$ is Boolean.

Proof As $S$ is the union of its finite sub-extensions, $S$ is reduced if and only if each finite sub-algebra is reduced, and all residue fields of $S$ are isomorphic to $k$ if and only if the same is true of any finite sub-algebra. Each finite sub-algebra $T$ is zero-dimensional, and hence a product of local rings. If $T$ is reduced, each factor is a field; if moreover each residue field is isomorphic to $k$, then $T \cong k^{n}$ (with $n \geq 2$ if $k \subset T$ ). Conversely, $k^{n}$ is reduced and all its residue fields are isomorphic to $k$. Finally, for each $x \in k^{n}$ (and thus for each $x \in S$ ), there is $a \in k^{n}$, such that $x=a x^{2}$. If $k=\mathbb{F}_{2}$, each $x \in k^{n}$ is such that $x=x^{2}$.

The canonical decomposition Considering an integral extension $k \subset S$, recall the canonical decomposition

$$
k \subseteq{ }_{S}^{+} k \subseteq{ }_{S}^{t} k \subseteq S,
$$

where ${ }_{S}^{+} k$ is the seminormalization of $k$ in $S$ and ${ }_{S}^{t} k$ its t-closure. We derive from Corollary 4.10 that either ${ }_{S}^{+} k={ }_{S}^{t} k$ or the closures are pushed to the extremities of the canonical decomposition.

Corollary 4.13 Let $k$ be a field and $k \subset S$ be a pointwise minimal extension. Then, either $k={ }_{S}^{+} k \subset{ }_{S}^{t} k=S$, in case the extension is decomposed, or ${ }_{S}^{+} k={ }_{S}^{t} k$. Moreover,

(1) if $k={ }_{S}^{+} k={ }_{S}^{t} k$, the extension is inert,

(2) if $S={ }_{S}^{+} k={ }_{S}^{t} k$, the extension is ramified,

(3) if $k \subset{ }_{S}^{+} k={ }_{S}^{t} k \subset S$, the extension is composite.

Proof If $k \subset S$ is decomposed, it follows from Proposition 4.11 that $k \subset S$ is seminormal, thus $k={ }_{S}^{+} k$, and infra-integral, thus ${ }_{S}^{t} k=S$. In all other cases, $S$ is a local ring (or a field) with maximal ideal $N$.

(1) If $k \subset S$ is inert, then $S$ is a field. Thus, $k \subset S$ is t-closed and $k={ }_{S}^{t} k$, a fortiori $k={ }_{S}^{+} k$. 
(2) If $k \subset S$ is ramified, then $S=k+N$. It follows that the extension is subintegral, thus ${ }_{S}^{+} k=S$, a fortiori ${ }_{S}^{t} k=S$.

(3) For the composite type, $k \subset k+N \subset S$. As for the ramified case, $k \subset k+N$ is subintegral (a fortiori also infra-integral). We show that $k+N \subset S$ is t-closed (a fortiori also seminormal). The rings $k+N$ and $S$ share the maximal ideal $N$. All properties of the extension $k+N \subset S$ are thus reflected in the residual extension $k \subset S / N$ (Proposition 2.13). As $S / N$ is a field, $k \subset S / N$ is t-closed. Thus, ${ }_{S}^{+} k={ }_{S}^{t} k=k+N$.

\subsection{Characterizations}

We characterize pointwise minimal extensions and pointwise minimal pairs of each type.

Decomposed type We first assume that $S$ is not a local ring: if $k \subset S$ is pointwise minimal, it is decomposed.

Proposition 4.14 Let $k \subset S$ be an integral extension over a field such that $S$ is not a local ring.

(1) $k \subset S$ is a pointwise minimal extension if and only if $S \cong k^{2}$ (that is, $k \subset S$ is minimal) or $k=\mathbb{F}_{2}$ and $S$ is Boolean.

(2) $k \subset S$ is a pointwise minimal pair if and only if $S \cong k^{2}$, or $k=\mathbb{F}_{2}$ and $S \cong k^{3}$.

Proof (1) By Propositions 4.11 and 4.12, if $k \subset S$ is pointwise minimal then each finite sub-extension is isomorphic to $k^{n}$ for some $n \geq 2$. This necessary condition turns out to be sufficient when $k=\mathbb{F}_{2}$. Indeed, in that case, $S$ is Boolean and each $x \in S$ is an idempotent. We now assume that $k \neq \mathbb{F}_{2}$. By way of contradiction, we suppose that $k \subset S$ is pointwise minimal and $\operatorname{dim}_{k}(S)>2$. The $k$-algebra generated by 3 linearly independent vectors of $S$ is finite, and hence isomorphic to $k^{n}$, for some $n \geq 3$. For each $m \leq n$, $k^{n}$ contains a sub- $k$-algebra isomorphic to $k^{m}$; in particular, $S$ contains a sub- $k$-algebra isomorphic to $k^{3}$. We show that $k \subset k^{3}$ is not pointwise minimal and thus reach a contradiction. As $k \neq \mathbb{F}_{2}$, there is $\lambda \in k$ distinct from 0 and 1 . Set $x:=(1, \lambda, 0)$. An elementary determinant computation shows that $\left(1, x, x^{2}\right)$ is a basis of the $k$-vector space $k^{3}$ :

$$
\left|\begin{array}{lll}
1 & 1 & 1 \\
1 & \lambda & 0 \\
1 & \lambda^{2} & 0
\end{array}\right|=\lambda^{2}-\lambda \neq 0
$$

Thus, $k[x]=k^{3}$, and $k \subset k[x]$ is not minimal. We reach a contradiction. Thus, in case $k \neq \mathbb{F}_{2}$, if $k \subset S$ is pointwise minimal, $\operatorname{dim}_{k}(S)=2$, that is, $k \subset S$ is minimal. Conversely, a minimal extension is obviously pointwise minimal.

(2) If $S \cong k^{2}$ (that is, $k \subset S$ is minimal), then $k \subset S$ is a pointwise minimal pair. In case $k=\mathbb{F}_{2}$ and $k \subset S$ is a pointwise minimal pair, then $k \subset S$ is a fortiori a pointwise minimal extension and $S$ is Boolean. If $S \cong k^{3}$, then $\operatorname{dim}_{k}(S)=3$ and thus $\ell[k, S]=2$. By Proposition 3.9, it follows that $k \subset S$ is a pointwise minimal pair. Finally, we show that, if $\operatorname{dim}_{k}(S)>3$, then $k \subset S$ is not a pointwise minimal pair. As above, it is enough to show that $k^{4}$ is not a pointwise minimal pair. Let $\left(e_{1}, \ldots, e_{4}\right)$ be the standard basis of $k^{4}$. Set $x:=e_{1}+e_{2}$, $T:=k[x]$, and $y:=e_{2}+e_{3}$. As $x y=e_{2}, x+x y=e_{1}$, and $y+x y=e_{3}, T[y]=k[x, y]$ contains the basis $\left(1, e_{1}, e_{2}, e_{3}\right)$ of $k^{4}$. As $t^{2}=t$ for each $t \in S$, it follows that $k[x]=k+k x$ and $k[x, x y]=k+k x+k x y$. Thus, $\operatorname{dim}_{k}(k[x])=2, \operatorname{dim}_{k}(k[x, y])=3$, and

$$
T=k[x] \subset k[x, x y] \subset k[x, y]=T[y]=k^{4} .
$$

Thus, $T \subset T[y]$ is not minimal.

Inert type We now turn to the inert type: $k \subset S$ is an algebraic field extension. Pointwise minimal extensions over a field are characterized in [4]. We easily add the consideration of pointwise minimal pairs.

Proposition 4.15 Let $k \subset S$ be an algebraic field extension. The following statements are equivalent:

(1) $k \subset S$ is either a minimal separable field extension, or $\operatorname{char}(k)=p$ and $S^{[p]} \subseteq k$,

(2) $k \subset S$ is a pointwise minimal extension,

(3) $k \subset S$ is a pointwise minimal pair. 
Proof (1) and (2) are equivalent by the characterization in [4, Proposition 4.16]. As a pointwise minimal pair is always a pointwise minimal extension, it remains to prove that (1) implies (3). If $k \subset S$ is minimal, there is no more to say. Otherwise, let $k \subset T$ be a sub-extension. Then, $T$ is a field. As $S^{[p]} \subseteq k$ obviously implies $S^{[p]} \subseteq T, T \subset S$ is pointwise minimal by (1).

Ramified type We next turn to the case where $S$ is a local ring with maximal ideal $N$ and $S=k+N$.

Proposition 4.16 Let $k \subset S$ be an integral extension over a field such that $S$ is a local ring with maximal ideal $N$ and $S=k+N$.

(1) $k \subset S$ is a pointwise minimal extension if and only if $N^{[2]}=(0)$.

(2) $k \subset S$ is a pointwise minimal pair if and only if $N^{2}=(0)$.

Proof (1) By Proposition 4.11, the condition $N^{[2]}=(0)$ is necessary. Conversely, if $x \in S$, then $x=a+n$, $a \in k, n \in N$, and thus $k[x]=k[n]$. If $x$ is not trivial, the condition $n^{2}=0$ implies $k[n] \cong k[X] /\left(X^{2}\right)$.

(2) Assume that $k \subset S$ is a pointwise minimal pair. Let $x, y \in N$. By (1), $x^{2}=y^{2}=0$, and to prove that $N^{2}=(0)$, it remains to show that $x y=0$. We consider two cases. If $k[x]=k[y]$, equivalently, $k x=k y$, then $x=a y$, with $a \in k$. Hence, $x y=a y^{2}=0$. Otherwise, $k[x] \subset k[x, y]$ is a minimal (integral) extension. The maximal ideal of $k[x]$ is $k x$, hence $x y \in k x$. With the same argument, $x y \in k y$. As $k x$ and $k y$ are vector spaces of dimension 1 and $k x \neq k y$, it follows that $k x \cap k y=(0)$. Therefore, $x y=0$.

Conversely, assume that $N^{2}=(0)$. Observe that, as $S=k+N$, it follows that each nontrivial $x \in S$ is of degree 2. Let $K \subset T$ be a sub-extension and $x \in S \backslash T$. We can choose $x \in N$. We show that $T \subset T[x]$ is minimal. For this, we show that, for each $t \in T[x]$, either $T[t]=T$ or $T[t]=T[x]$. As $x$ is of degree 2, a fortiori $T[x]=T+T x$; thus, one can write $t=a+b x$, with $a, b \in T$. If $b \in N$, then $b x \in N^{2}$. Thus, $b x=0$, and $T[t]=T$. If $b \notin N, b$ is a unit in $T$, and thus $x=b^{-1}(t-a)$, and $T[t]=T[x]$.

In fact, one derives from next lemma (inspired by [4, Theorem 4.9]) that, unless $\operatorname{char}(k)=2$, pointwise minimal ramified extensions are pointwise minimal pairs.

Lemma 4.17 Let $k$ be a field of characteristic char $(k) \neq 2$ and I be an ideal of a $k$-algebra S. Then, $I^{[2]}=(0)$ if and only if $I^{2}=(0)$.

Proof If $I^{[2]}=(0)$, the relation $(x+y)^{2}=x^{2}+2 x y+y^{2}$ brings $2 x y=0$. If $\operatorname{char}(k) \neq 2$, it follows that $I^{2}=(0)$. The converse is obvious.

In the last section, we give examples of pointwise minimal extensions that are not pointwise minimal pairs (Example 6.1). By the previous lemma, $\operatorname{char}(k)=2$. We also give examples of pointwise minimal pairs over fields of any characteristic (Example 6.2).

Composite type We finally consider the case where $S$ is a local ring with maximal ideal $N$ and $k \subset k+N \subset S$. By Corollary 4.10, some minimal elements are ramified (those in $k+N$ ), while the others are inert.

Proposition 4.18 Let $k \subset S$ be an integral extension over a field such that $S$ is a local ring with maximal ideal $N$ and $k \subset k+N \subset S$. Then, $k \subset S$ is a pointwise minimal extension if and only if $\operatorname{char}(k)=p, N^{[2]}=(0)$ and $S^{[p]} \subseteq k$. Such an extension is never a pointwise minimal pair.

Proof All conditions are necessary by Proposition 4.11. Conversely, assume these conditions and let $x \in S$. Then, $x^{p}=a \in k$. As necessarily $\operatorname{char}(k)=p$, the polynomial $P=X^{p}-a$ is either irreducible in $k[X]$, or such that $P=(X-b)^{p}$, with $b \in k$. In the first case, $k \subset k[y]$ is a minimal field extension: $y$ is inert. In the second case, $(x-b)^{p}=0$ and $(x-b)$ is nilpotent. If $x=b$, then $x$ is trivial. Otherwise, the condition $N^{[2]}=(0)$ implies that $x$ is minimal ramified.

Finally, we show that, if $x$ is nilpotent of degree 2 and $y$ is inert, then $k[x] \subset k[x, x y] \subset k[x, y]=k[x][y]$, and hence that $k \subset S$ is not a pointwise minimal pair. Clearly, $x, x y \in N$, thus $k[x, x y] \subseteq k+N$. Therefore, $k[x, x y] \subset k[x, y]$. By way of contradiction, suppose that $x y \in k[x]=k+k x$. As $x y \in N, x y=a x$, with $a \in k$. Hence, $x(y-a)=0$. As $k[y]$ is a field, and $y \notin k,(y-a)$ is a unit. Hence, $x=0$ and we reach a contradiction. Therefore, $k[x] \subset k[x, x y]$.

Remark 4.19 Clearly, $S^{[p]} \subseteq k$ implies $(S / N)^{[p]} \subseteq k$. However, one cannot replace $S^{[p]} \subseteq k$ by $(S / N)^{[p]} \subseteq k$ in Proposition 4.18 (see Example 6.5). In fact, a composite pointwise minimal extension is such that $S=L+N$, where $L$ is a field (isomorphic to $S / N$ ). When this is the case (contrary to Example 6.5), one can replace $S^{[p]} \subseteq k$ by $L^{[p]} \subseteq k$.

The following alternate characterization shows that composite pointwise minimal extensions effectively occur (see Example 6.4). 
Proposition 4.20 An extension $k \subset S$ over a field is a composite pointwise minimal extension if and only if $\operatorname{char}(k)=p$ and $S=L+N$, where $L$ is a proper field extension of $k$ such that $L^{[p]} \subseteq k$, and $N$ is a non-zero ideal of $S$ such that $N^{[2]}=(0)$.

Proof Assume that $\operatorname{char}(k)=p$ and $S=L+N$, with $L$ and $N$ as needed. As $N \neq(0)$ and $k \subset L$, it follows that $k \subset k+N \subset S=L+N$. Finally, let $y \in S$, and write $y=b+n, b \in L, n \in N$. Then, $L^{[p]} \subseteq k$ and $n^{2}=0$ bring $y^{p}=b^{p}+n^{p}=b^{p} \in k$. Thus, $S^{[p]} \subseteq k$ and all conditions of Proposition 4.18 are met.

Conversely, if $k \subset S$ is a composite pointwise minimal extension, then $\operatorname{char}(k)=p, S^{[p]} \subseteq k, S$ is a local ring with maximal ideal $N$, and $N^{[2]}=(0)$. It thus remains to show there is a field $L$ such that $k \subset L \subset S$ and $S=L+N$. Consider the set $\mathcal{K}$ of fields $K$ such that $k \subseteq K \subset S$. Allowing $k$ itself, this set is not empty. By Zorn's lemma ( or trivially if $k \subset S$ is finite), every element of $\mathcal{K}$ is contained in a maximal element. Let $L$ be maximal in $\mathcal{K}$. We claim that $S=L+N$. If not, we have $L \subset L+N \subset S$. As $N^{[2]}=(0)$, and $S^{[p]} \subseteq k$ (a fortiori $S^{[p]} \subseteq L$ ), it follows from Proposition 4.18 that $L \subset S$ is a composite pointwise minimal extension. In particular, there is an inert element $y \in S$. Thus, $L[y]$ is a field and $L \subset L[y] \subset S$. We reach a contradiction.

Remark 4.21 The field $L$ is not unique. Pick $a \in L, n \in N$, with $a \notin k, n \neq 0$, and set $x:=a+n$. Then $x \notin k+N$, thus $k[x]$ is a field, clearly not contained in $L$. As above, $k[x]$ is contained in a maximal element $L^{\prime}$ of $\mathcal{K}$. Then, $S=L^{\prime}+N$, with $L^{\prime} \neq L$. Yet, $L \cong L^{\prime}$, as both are isomorphic to $S / N$.

\section{General integral extensions}

We now harvest the results of the previous section in the general context of integral extensions.

\subsection{The four types}

We derive from Corollary 4.10 that there are four types of pointwise minimal integral extensions. We already gave them a name (Definition 4.9).

Proposition 5.1 Let $R \subset S$ be a pointwise minimal integral extension with crucial ideal $M$.

(1) If there is more than one maximal ideal of $S$ above $M$, then all minimal sub-extensions are decomposed.

(2) If there is only one maximal ideal $N$ of $S$ above $M$, then all minimal sub-extensions are either ramified or inert. More precisely, $R \subset S$ is inert if and only if $S / M$ is a field, ramified if and only if $S=R+N$, and composite if and only if $R \subset R+N \subset S$.

In the composite case, a minimal element $x \in S$ is ramified if $x \in R+N$, and otherwise inert.

Proof An element $x \in S$ is minimal (resp. trivial) if and only if its class $\bar{x}$ modulo $M$ is minimal (resp. trivial) in the extension $R / M \subset S / M$ (Proposition 2.8). Moreover, the type (decomposed, inert, or ramified) of $x$ is (by definition) the same as the type of $\bar{x}$. The fact that $x \in S$ is ramified if and only if $x \in R+N$ follows from Proposition 4.5 .

In fact, not only the minimal extensions $R \subset R[x]$ are of the appropriate type, but also all intermediate minimal extensions, in particular each step of a saturated chain from $R$ to $S$ (if any).

Corollary 5.2 Let $R \subset S$ be a pointwise minimal integral extension and let $T \subset T^{\prime}$ be a minimal extension such that $R \subseteq T \subset T^{\prime} \subseteq S$. If $R \subset S$ is decomposed (resp. inert, resp. ramified), then $T \subset T^{\prime}$ is decomposed (resp. inert, resp. ramified). If $R \subset S$ is composite, then $T \subset T^{\prime}$ is either ramified or inert.

Proof We let $M$ be the crucial ideal of the extension. We then have $R / M \subseteq T / M \subset T^{\prime} / M \subseteq S / M$, and $T / M \subset T^{\prime} / M$ is minimal, of the same type as $T \subset T^{\prime}$.

(a) If $S / M$ is a field, so are $T / M$ and $T^{\prime} / M$, and that settles the inert type.

(b) If $S / M$ is a (zero-dimensional) local ring, so are $T / M$ and $T^{\prime} / M$, and thus $T / M \subset T^{\prime} / M$ is not decomposed. That settles the composite type.

(c) If all residue fields of $S / M$ are isomorphic to $R / M$, so are all residue fields of $T / M$ and $T^{\prime} / M$. Thus $T / M \subset T^{\prime} / M$ is not inert.

(d) If $S / M$ is a reduced ring, so are $T / M$ and $T^{\prime} / M$; thus, $T / M \subset T^{\prime} / M$ is not ramified.

Apply (c) and (d) for the decomposed type, and (b) and (c) for the ramified type. 
Characterization Let $R \subset S$ be a pointwise minimal integral extension with crucial ideal $M$. If there is only one maximal ideal $N$ of $S$ above $M$, then $S / M$ is a local ring. By Proposition 4.11, $(N / M)^{[2]}=(0)$, equivalently $N^{[2]} \subseteq M$. We discuss this condition in the following lemma. This leads to simpler formulations in the characterization of ramified pointwise minimal extensions (and pairs), generalizing [4, Proposition 4.11], with no restriction to the local case.

Lemma 5.3 Let $R \subset S$ be an integral extension with a maximal ideal $M$ of $R$ such that $M S=M$. The following assertions are equivalent.

(1) there is only one maximal ideal $N$ of $S$ above $M, S=R+N$, and $N^{[2]} \subseteq M$ (resp. $N^{2} \subseteq M$ ),

(2) there is an ideal $I$ of $S$ such that $S=R+I$, and $I^{[2]} \subseteq M$ (resp. $I^{2} \subseteq M$ ).

Proof Assume there is an ideal $I$ of $S$ such that $S=R+I$. Set $N:=I+M$. Clearly, $S=R+N$. As $S / N=(R+N) / N \cong R /(R \cap N)=R / M$, it follows that $N$ is a maximal ideal of $S$. For $n \in N$, write $n=i+m, i \in I, m \in M$. If $I^{[2]} \subseteq M$, then $n^{2}=i^{2}+2 i m+m^{2} \in M$ (using the fact that $M S=M$ ). Thus, $I^{[2]} \subseteq M$ implies $N^{[2]} \subseteq M$. Considering the product of a pair of elements in $N$, a similar argument shows that $I^{2} \subseteq M$ implies $N^{\frac{1}{2}} \subseteq M$. Finally, $N^{[2]} \subseteq M$ implies that $N$ is the only maximal ideal of $S$ above $M$. Thus, (2) implies (1). The converse is obvious

Theorem 5.4 Let $R \subset S$ be an integral extension. Then, $R \subset S$ is a pointwise minimal extension if and only if there is a maximal ideal $M$ of $R$ such that $M S=M$ and, letting $k:=R / M$, one of the following mutually exclusive conditions is satisfied.

(1) Decomposed type: either $S / M \cong k^{2}$ or $k=\mathbb{F}_{2}$ and $S / M$ is Boolean.

(2) Inert type: $S / M$ is a field and either $k \subset S / M$ is a separable minimal field extension or char $(k)=p$ and $S^{[p]} \subseteq R$.

(3) Ramified type: there is an ideal I of $S$ such that $S=R+I$, and $I^{[2]} \subseteq M$.

(4) Composite type: there is only one maximal ideal $N$ of $S$ above $M, R \subset R+N \subset S, N^{[2]} \subseteq M$, $\operatorname{char}(k)=p$ and $S^{[p]} \subseteq R$.

Proof The decomposed case derives immediately from Proposition 4.14, the ramified case from Lemma 5.3 and Proposition 4.16, and finally the inert and composite cases from Propositions 4.15 and 4.18 and the fact that, as $M S=M,(S / M)^{[p]} \subseteq R / M$ if and only if $S^{[p]} \subseteq R$.

Remark 5.5 (1) The hypothesis there is a maximal ideal $M$ of $R$ such that $M S=M$ is essential (see the case of polynomials in Sect. 6.2).

(2) Let $R \subset S$ be a pointwise minimal extension and $J:=\sqrt[S]{M}$ be the intersection of the maximal ideals of $S$ above $M$. If there is only one maximal ideal $N$ of $S$ above $M$, then $J=N$. Otherwise, $R \subset S$ is decomposed and $S / M$ is reduced, thus $J=M$. In any case, $J^{[2]} \subseteq M$. This is [4, Corollary 4.10] (in the context of domains).

(3) In the decomposed case (and that case only), there is more than one maximal ideal above $M$ in $S$. If $k \neq \mathbb{F}_{2}$, the extension is minimal and there are only two such ideals, and if $k=\mathbb{F}_{2}$, these ideals can be in any number, even infinitely many.

(4) A pointwise minimal extension with an ideal $I$ such that $I^{[2]} \subseteq M$ and $R \subset R+I \subset S$, need not be composite: if $N$ is the single maximal ideal of $S$ above $M$, one can have $R+I \subset R+N$ and then, either $S=R+N$ (the extension is ramified) or $R+N \subset S$ (the extension is composite).

(5) An integrally closed pointwise minimal extension is in fact minimal (Proposition 3.5). In the integral case, we can conclude similarly in two cases, just by considering the residue field $k:=R / M$ :

- if $k \neq \mathbb{F}_{2}$, a decomposed pointwise minimal extension is minimal,

- if $k$ is a perfect field (in particular, if $\operatorname{char}(k)=0$ or $k$ is finite), an inert pointwise minimal extension is minimal.

On the other hand, a pointwise minimal extension of the composite type is obviously never minimal.

Pointwise minimal pairs From Proposition 4.15 a pointwise minimal extension is always a pointwise minimal pair in the inert case, never in the composite case from Proposition 4.18. The decomposed and ramified cases deserve more details. We summarize this situation as follows.

Proposition 5.6 Let $R \subset S$ be an integral extension. Then, $R \subset S$ is a pointwise minimal pair if and only if there is a maximal ideal $M$ of $R$ such that $M S=M$ and, letting $k:=R / M$, one of the following mutually exclusive conditions is satisfied. 
(1) Decomposed type: either $S / M \cong k^{2}$ or $k=\mathbb{F}_{2}$ and $S / M \cong k^{3}$.

(2) Inert type: same condition as for pointwise minimal extensions (Theorem 5.4).

(3) Ramified type: there is an ideal I of $S$ such that $S=R+I$, and $I^{2} \subseteq M$.

(4) Composite type: there are no pointwise minimal pairs of the composite type.

Proof The decomposed case follows immediately from Proposition 4.14, and the ramified case from Proposition 4.16 and Lemma 5.3.

Aside from minimal extensions and pointwise minimal inert extensions, pointwise minimal extensions need not be pointwise minimal pairs. In the ramified type, we however have the following (inspired by [4, Theorem 4.9]).

Corollary 5.7 Let $R \subset S$ be an integral pointwise minimal ramified extension with crucial maximal ideal $M$. If either $\operatorname{char}(R / M) \neq 2$ or there is $x \in S$ such that $x^{2} \in M$ and $S=R+S x$, then $R \subset S$ is a pointwise minimal pair.

Proof Let $N$ be the single maximal ideal of $S$ above $M$. As $R \subset S$ is a pointwise minimal ramified extension, $N^{[2]} \subseteq M$. If $\operatorname{char}(R / M) \neq 2$, it follows from Lemma 4.17 that $N^{2} \subseteq M$. If there is $x \in S$ such that $x^{2} \in M$ and $S=R+S x$, let $I:=S x$. Clearly, $I^{2} \subseteq M$. In both cases, $R \subset S$ is a pointwise minimal pair by Proposition 5.6.

\subsection{Chain conditions}

Pointwise minimal extensions have FCP, or FMC if and only if they have finite length (Proposition 3.6). We now give length formulae for all cases, but the composite type (we next devote a whole subsection to this case).

Proposition 5.8 Let $R \subset S$ be a finite pointwise minimal extension with crucial ideal $M$ and $k:=R / M$. Set $\ell:=\ell[R, S]$, then

(1) If $R \subset S$ is decomposed, then $\ell=\operatorname{dim}_{k}(S / M)-1$.

(2) If $R \subset S$ is ramified and $N$ is the single maximal ideal of $S$ above $M$, then $\ell=\operatorname{dim}_{k}(S / M)-1=$ $\operatorname{dim}_{k}(N / M)$.

(3) If $R \subset S$ is inert, then either $k \subset S / M$ is separable and $\ell=1$, or $\operatorname{char}(k)=p$ and $p^{\ell}=[S / M: k]$.

Moreover, in each case, all saturated chains from $R$ to $S$ have length $\ell$.

Proof Considering the residual extension $k:=R / M \subset S / M$, let

$$
k=R_{0} \subset R_{1} \subset \cdots R_{i} \subset R_{i+1} \subset \cdots R_{n}=S / M
$$

be a saturated chain from $k$ to $S / M$. By Corollary 5.2, all minimal extensions $R_{i} \subset R_{i+1}$ are of the same type. Let $M_{i}$ be the crucial ideal of the minimal extension $R_{i} \subset R_{i+1}$.

In case $k \subset S / M$ is either decomposed or ramified, all residue fields of $S / M$ are isomorphic to $k$, a fortiori $R_{i} / M_{i} \cong k$. Then, $R_{i+1} / M_{i} \cong k^{2}$ in the decomposed case and $R_{i+1} / M_{i} \cong k[X] /\left(X^{2}\right)$ in the ramified case. In both cases, $\operatorname{dim}_{k}\left(R_{i+1} / M_{i}\right)=\operatorname{dim}_{k}\left(R_{i} / M_{i}\right)+1$. By elementary linear algebra, it follows that $\operatorname{dim}_{k}\left(R_{i+1}\right)=\operatorname{dim}_{k}\left(R_{i}\right)+1$. As $\operatorname{dim}_{k}\left(R_{0}\right)=1$, and we thus have $\operatorname{dim}_{k}(S / M)=n+1$. The length of every saturated chain is then $\ell=\operatorname{dim}_{k}(S / M)-1$. Moreover, in the ramified case, $S=R+N$, thus $S / M=k+N / M$, and hence $\operatorname{dim}_{k}(S / M)=\operatorname{dim}_{k}(N / M)+1$.

In case $k \subset S / M$ is inert, it follows by Proposition 4.15 that either $k \subset S / M$ is a minimal field extension or $(S / M)^{[p]} \subset k$. In the first case, $\ell[k, S / M]=1$. In the second, each step $R_{i} \subset R_{i+1}$ is a minimal inseparable field extension, hence $\left[R_{i+1}: R_{i}\right]=p$, and thus $[S / M: k]=p^{n}$.

Co-pointwise minimal extensions Co-pointwise minimal extensions are but pointwise minimal pairs of length 2 (Proposition 3.9):

Corollary 5.9 Let $R \subset S$ be an integral extension. Then $R \subset S$ is a co-pointwise minimal extension if and only if there is a maximal ideal $M$ of $R$ such that $M S=M$ and one of the following mutually exclusive conditions is satisfied, where $k:=R / M$.

(1) Decomposed type: $k=\mathbb{F}_{2}$ and $S / M \cong k^{3}$.

(2) Inert type: $S / M$ is a field, $(S / M)^{[p]} \subseteq k$, and $[S / M: k]=p^{2}$. 
(3) Ramified type: $S / M \cong k[X, Y] /\left(X^{2}, X Y, Y^{2}\right)$.

Proof Among pointwise minimal pairs (characterized in Proposition 5.6), we must look at those of length 2, in particular, discard minimal extensions. In the decomposed case, we are left with $S / M \cong k^{3}$. In the inert case, we are left with radicial field extensions such that, by Proposition 5.8, $[S / M: k]=p^{2}$. In the ramified case, we use the fact that, by Proposition 3.9, $S / M=k[x, y]$ with $(x, y)$ a minimal system of two generators. As $S / M=k+N$, we may as well assume that $x, y \in N$. As $N^{2}=(0), x^{2}=y^{2}=x y=0$. Therefore, $S / M=k[x, y]$ is isomorphic to a quotient of $T:=k[X, Y] /\left(X^{2}, X Y, Y^{2}\right)$. Finally, as $\operatorname{dim}_{k}(T)=3$, one must have $S / M \cong k[X, Y] /\left(X^{2}, X Y, Y^{2}\right)$. Conversely, in each case, it is easy to see that $k \subset S / M$, and then also $R \subset S$, is of length 2 .

FIP extensions Unless it is minimal, a pointwise minimal extension $R \subset S$ with crucial ideal $M$ has FIP if and only if the extension is finite and $R / M$ is a finite field (Proposition 3.7). Examples 6.1 and 6.2 provide rather large families of pointwise minimal ramified extensions with FIP. The other cases are more restrictive.

Corollary 5.10 Let $R \subset S$ be an integral pointwise minimal extension with crucial ideal $M$ and $k:=R / M$.

(1) In the decomposed case, $R \subset S$ has FIP if and only if either $S / M \cong k^{2}$ or $k=\mathbb{F}_{2}$ and $S / M \cong k^{n}$, $n \geq 2$.

(2) In the inert case, $R \subset S$ has FIP if and only if it is a minimal extension.

(3) In the composite case, $R \subset S$ never has FIP.

Proof In the decomposed case, unless $R \subset S$ is minimal, then $k=\mathbb{F}_{2}$, and the extension has FIP if and only if it is finite, that is, $S / M \cong k^{n}, n \geq 2$. In the inert case, if $R \subset S$ is not minimal, then $k \subset S / M$ is not separable and $k$ is not a finite field. In the composite case, $R \subset S$ is never minimal and $k$ always infinite, and thus $R \subset S$ never has FIP.

Remark 5.11 Comparing Corollary 5.9 with the previous result, it appears that every decomposed co-pointwise minimal extension has FIP but no inert ones. On the other hand, a ramified co-pointwise minimal extensions has FIP if and only if the residue field $k:=R / M$ is finite (and there are no composite ones).

\subsection{More on the composite type}

Hypotheses and notations In this subsection, $R \subset S$ is a composite pointwise minimal extension with crucial maximal ideal $M, k:=R / M, \operatorname{char}(k)=p$, and $N$ denotes the only maximal ideal of $S$ above $M$. We let $T:=R+N$ and (by Proposition 4.20) $L$ be a field such that $S / M=L+N / M$.

Good rings between $R$ and $S$ A composite pointwise minimal extension is never a pointwise minimal pair. We discuss which rings $R^{\prime}$ between $R$ and $S$ are such that $R^{\prime} \subset S$ is pointwise minimal.

Proposition 5.12 Let $R^{\prime}$ be a ring between $R$ and $S$ and $M^{\prime}:=N \cap R^{\prime}$. Then, $R^{\prime} \subset S$ is a pointwise minimal extension if and only if $M^{\prime} S=M^{\prime}$. In that case, $R^{\prime} \subset S$ is inert if and only if $R^{\prime}=R^{\prime}+N$, ramified if and only if $R^{\prime}+N=S$, and composite if and only if $R^{\prime} \subset R^{\prime}+N \subset S$.

Proof As $N$ is the only maximal ideal of $S$ above $M, M^{\prime}$ is the only maximal ideal of $R^{\prime}$ above $M$. Every other prime $P^{\prime}$ of $R^{\prime}$ is over some prime $P$ distinct from $M$. Then, $R_{P}=S_{P}$, and thus $R_{P^{\prime}}^{\prime}=S_{P^{\prime}}$. Hence, $M^{\prime}$ is the crucial ideal of the extension $R^{\prime} \subset S$. By Theorem 3.2, if $R^{\prime} \subset S$ is pointwise minimal, then $M^{\prime} S=M^{\prime}$. Conversely, assume that $M^{\prime} S=M^{\prime}$. As $N^{[2]} \subseteq M \subseteq M^{\prime}$, and $S^{[p]} \subseteq R \subseteq R^{\prime}$, all conditions of Theorem 5.4 are met to conclude that $R^{\prime} \subset S$ is a pointwise minimal extension, in each three possible cases: $R^{\prime}=R^{\prime}+N$, then $R^{\prime} \subset S$ is inert (since in that case $M^{\prime}=N$ and $S / N$ is a field), $R^{\prime}+N=S$, then $R^{\prime} \subset S$ is ramified, or $R^{\prime} \subset R^{\prime}+N \subset S$, then $R^{\prime} \subset S$ is composite.

We derive two easy cases where $R^{\prime} \subset S$ is pointwise minimal.

Corollary 5.13 Let $R^{\prime}$ be a ring between $R$ and $S$.

(1) If $T \subseteq R^{\prime}$, then $R^{\prime} \subset S$ is a pointwise minimal extension of the inert type.

(2) If $R^{\prime} / M$ is a field, then $R^{\prime} \subset S$ is a pointwise minimal extension. Moreover, $R^{\prime} \subset S$ is ramified if $R^{\prime} / M \cong S / N$, otherwise composite. 
Proof (1) If $T:=R+N \subseteq R^{\prime}$, then $R^{\prime} \cap N=N$. As $N$ is an ideal of both $R^{\prime}$ and $S$, it is clear that $N S=N$. By Proposition 5.12, $R \subset S$ is pointwise minimal. As obviously $R^{\prime}+N=R^{\prime}, R^{\prime} \subset S$ is inert.

(2) If $R^{\prime} / M$ is a field, $M$ is maximal in $R^{\prime}$, and hence $R^{\prime} \cap N=M$. As $M S=M, R^{\prime} \subset S$ is pointwise minimal. As $M$ is maximal in $R^{\prime}$, not in $R^{\prime}+N$, it follows that $R^{\prime} \subset R^{\prime}+N$. Thus, $R^{\prime} \subset S$ is either ramified or composite. It is ramified, if and only if $S=R^{\prime}+N$. As $S$ and $R^{\prime}+N$ share the ideal $N$, this is the case if and only if $S / N=\left(R^{\prime}+N\right) / N$, that is, if and only if $S / N \cong R^{\prime} /\left(R^{\prime} \cap N\right)=R^{\prime} / M$.

We next show how to obtain rings $R^{\prime}$ such that $R^{\prime} / M$ is a field.

Proposition 5.14 For each field $K$ such that $k \subseteq K \subseteq L$, there is a ring $R^{\prime}$ between $R$ and $S$ such that $R^{\prime} / M \cong K$.

Proof As $k=R / M \subseteq K \subseteq L \subset S / M$, there is a unique ring $R^{\prime}$ between $R$ and $S$ such that $R^{\prime} / M=K$.

Remark 5.15 The proof above does not say there is a unique ring $R^{\prime}$ such that $R^{\prime} / M \cong K$. In particular, by Remark 4.21, there is a field $L^{\prime}$, distinct from $L$, such that $S / M=L^{\prime}+N / M$. There is a unique ring $R_{1}$ such that $R_{1} / M=L$ and a unique $R_{2}$ such that $R_{2} / M=L^{\prime}$. These rings are distinct, yet both $R_{1} / M$ and $R_{2} / M$ are isomorphic to $S / N$.

More hypotheses and notations From then on, we assume that $R \subset S$ is finite, equivalently that $\ell:=\ell[R, S]$ is finite, or that $\operatorname{dim}_{k}(S / M)$ is finite (Proposition 3.6).

As $S / M=L+N / M$, we can consider $N / M$ as an $L$-vector space. We set $\alpha:=\operatorname{dim}_{L}(N / M)$ and let $\ell_{1}$ be the integer such that $[L: k]=p^{\ell_{1}}$.

Length of chains We show there is a chain of maximal length that goes through $T$ and gives a formula for $\ell[R, S]$. Recall that, by Corollary 5.2, each step of a saturated chain is either inert or ramified.

Proposition 5.16 (1) For each saturated chain from $R$ to $S$,

(a) the number of inert steps is $\ell_{1}$,

(b) the number of ramified steps is bounded by $\ell[R, T]$.

(2) $\ell[R, T]=\alpha p^{\ell_{1}}, \ell[T, S]=\ell_{1}$,

$$
\ell[R, S]=\ell[R, T]+\ell[T, S]=\alpha p^{\ell_{1}}+\ell_{1},
$$

the length of every saturated chain that goes through $T$ is $\ell[R, S]$.

Proof (1) Consider a saturated chain of length $n$,

$$
R=R_{0} \subset \cdots \subset R_{i} \subset R_{i+1} \subset \cdots \subset R_{n}=S .
$$

For each $i, M_{i}:=R_{i} \cap N$ is the only maximal ideal of $R_{i}$ above $M$.

(a) Setting $K_{i}:=R_{i} / M_{i}$, we obtain a chain of fields:

$$
k=K_{0} \subseteq \cdots \subseteq K_{i} \subseteq K_{i+1} \subseteq \cdots \subseteq K_{n}=L .
$$

Each step $R_{i} \subset R_{i+1}$ is a minimal extension. In case this step is inert, [ $K_{i+1}: K_{i}$ ] $=p$, in case it is ramified, $K_{i+1}=K_{i}$ and $\left[K_{i+1}: K_{i}\right]=1$. As $p^{\ell_{1}}=[L: k]=\prod_{i=0}^{n-1}\left[K_{i+1}: K_{i}\right]$, the number of inert steps is $\ell_{1}$.

(b) Setting $T_{i}=R_{i} \cap T$, we obtain a chain of rings from $R$ to $T$ :

$$
R=T_{0} \subseteq \cdots \subseteq T_{i} \subseteq T_{i+1} \subseteq \cdots \subseteq T_{n}=T .
$$

If $R_{i} \subset R_{i+1}$ is ramified, then $R_{i+1}=R_{i}\left[x_{i}\right]=R+R x_{i}$, and one can as well take $x_{i}$ such that $x_{i}^{2} \in M_{i}$. In particular, $x_{i} \in N$, a fortiori $x_{i} \in T=R+N$. Hence, $x_{i} \in T_{i+1}=R_{i+1} \cap T$. As $x_{i} \notin R_{i}$, a fortiori $x_{i} \notin T_{i}$, it follows that $T_{i} \subset T_{i+1}$. The number of ramified steps is therefore bounded by $\ell[R, T]$.

(2) By Corollary 5.13, $T \subset S$ is an inert pointwise minimal extension with crucial ideal $N, T / N \cong k$, and $S / N \cong L$. Also, $R \subset T=R+N$ is a ramified pointwise minimal extension, with crucial ideal $M$. Proposition 5.8 provides the length of both extensions:

- $\ell[T, S]=\ell_{1}$, since $[L: k]=p^{\ell_{1}}$,

- $\ell[R, T]=\operatorname{dim}_{k}(N / M)=\operatorname{dim}_{L}(N / M)[L: k]=\alpha p^{\ell_{1}}$. 
If a saturated chain goes through $T$, each step from $R$ to $T$ is ramified, and each step from $T$ to $S$ is inert. By Proposition 5.8, the number of inert steps is $\ell[T, S]$, and the number of ramified steps is $\ell[R, T]$. By (1) these numbers are maximal. The length of each such chain is thus

$$
\ell[R, S]=\ell[R, T]+\ell[T, S]=\alpha p^{\ell_{1}}+\ell_{1} .
$$

We now show there are chains of length $n<\ell[R, S]$.

Corollary 5.17 For each $i, 0 \leq i \leq \ell_{1}$, there is a saturated chain from $R$ to $S$ of length $n=\alpha p^{i}+\ell_{1}$.

Proof For each $i, 0 \leq i \leq \ell_{1}$, there is a field $K_{i}$ such that $k \subseteq K_{i} \subseteq L$ and $\left[L: K_{i}\right]=p^{i}$. By Proposition 5.14, there is a ring $R^{\prime}$ such that $R \subseteq R^{\prime} \subset S$ and $R^{\prime} / M \cong K_{i}$. By Corollary 5.13, $R^{\prime} \subset S$ is a pointwise minimal extension either ramified or composite. In any case, as $R^{\prime} / M$ is a field, the crucial ideal of the extension is $M$. If $R^{\prime} \subset S$ is composite, we use Proposition 5.16 to compute $\ell\left[R^{\prime}, S\right]$. As $S / N \cong L$, and $R^{\prime} / M \cong K_{i}$, the parameters to use are $\left[L: K_{i}\right]=p^{i}$, and $\alpha=\operatorname{dim}_{L}(N / M)$. Thus, $\ell\left[R^{\prime}, S\right]=\alpha p^{i}+i$. The same formula applies with $i=0$, if $R^{\prime} \subset S$ is ramified. Indeed, by Proposition 5.8, $\ell\left[R^{\prime}, S\right]=\operatorname{dim}_{L}(N / M)=\alpha$. On the other hand, $R^{\prime} \subset S$ is ramified if and only if $R^{\prime} / M \cong L$. In that case $i=0$.

As $R^{\prime} / M$ is a field, $R \subset R^{\prime}$ is an inert pointwise minimal extension. As $[L: k]=\left[L: K_{i}\right]\left[K_{i}: k\right]$, $\left[K_{i}: k\right]=p^{\ell_{1}-i}$. Hence, $\ell\left[R, R^{\prime}\right]=\ell_{1}-i$. Combining a saturated chain from $R$ to $R^{\prime}$ with a saturated chain from $R^{\prime}$ to $S$, we obtain a saturated chain of length

$$
\ell\left[R, R^{\prime}\right]+\ell\left[R^{\prime}, S\right]=\ell_{1}-i+\alpha p^{i}+i=\alpha p^{i}+\ell_{1} .
$$

Example 6.4 shows that $\alpha$ and $\ell_{1}$ can arbitrarily be chosen. The smallest possible values are $\alpha=1, \ell_{1}=1$ and $p=2$. In that case $\ell[R, S]=3$, and the shortest chains have length 2 .

Bad rings between $R$ and $S$ In Corollary 5.13, we gave sufficient conditions for a ring $R^{\prime}$ between $R$ and $S$ to be "good", that is, such that $R^{\prime} \subset S$ is pointwise minimal. We now give necessary conditions. It is easy to obtain "bad" rings which do not meet these conditions.

Corollary 5.18 Let $R^{\prime}$ be a ring between $R$ and $S$ such that $R^{\prime} / M$ is a field $K$ such that $[L: K]=p^{i}$, and $R_{1}$ be a ring between $R^{\prime}$ and $R^{\prime}+N$. If $R_{1} \subset S$ is pointwise minimal, then $p^{i}$ divides $\ell\left[R^{\prime}, R_{1}\right]$. In particular, if $x_{1}, \ldots, x_{n}$ are ramified elements of $S$ and $n<p^{\ell_{1}}$, then $R\left[x_{1}, \ldots, x_{n}\right] \subset S$ is not pointwise minimal.

Proof By Corollary 5.13, as $R^{\prime} / M$ is a field, $R^{\prime} \subset S$ is pointwise minimal. The proof of Corollary 5.17 shows that $\ell\left[R^{\prime}, S\right]=\alpha p^{i}+i$. Let $T^{\prime}:=R^{\prime}+N$. One has more precisely $\ell\left[R^{\prime}, T^{\prime}\right]=\alpha p^{i}$ (the number of ramified steps) and $\ell\left[T^{\prime}, S\right]=i$ (the number of inert steps). Suppose that $R_{1} \subset S$ is pointwise minimal. As $R^{\prime} \subseteq R_{1} \subseteq T^{\prime}=R^{\prime}+N$, it is clear that $T^{\prime}=R_{1}+N$. By Proposition 5.16 a chain of maximal length from $R_{1}$ to $S$ passes through $T^{\prime}$. As the number of inert steps is $\ell\left[T^{\prime}, S\right]=i$, it follows that the number of ramified steps is $\ell\left[R_{1}, T^{\prime}\right]=\alpha^{\prime} p^{i}$, for some positive integer $\alpha^{\prime}$. By Proposition 5.8, all saturated chains from $R^{\prime}$ to $T^{\prime}$ have same length, since $R^{\prime} \subset T^{\prime}=R^{\prime}+N$ is pointwise minimal of the ramified type. It follows that $\ell\left[R^{\prime}, T^{\prime}\right]=\ell\left[R^{\prime}, R_{1}\right]+\ell\left[R_{1}, T^{\prime}\right]$. As $p^{i}$ divides both $\ell\left[R^{\prime}, T^{\prime}\right]$ and $\ell\left[R_{1}, T^{\prime}\right]$, it also divides $\ell\left[R^{\prime}, R_{1}\right]$.

In particular, one can consider the case where $R^{\prime}=R$, and hence $R_{1}$ is between $R$ and $T=R+N$. It follows that if $R_{1} \subset S$ is pointwise minimal, then $p^{\ell_{1}}$ divides $\ell\left[R, R_{1}\right]$. If $x_{1}, \ldots, x_{n}$ are ramified, they all belong to $T$. Let $R_{1}:=R\left[x_{1}, \ldots, x_{n}\right]$. For each $i \leq n$, either $R\left[x_{1}, \ldots, x_{i-1}\right]=R\left[x_{1}, \ldots, x_{i-1}\right]\left[x_{i}\right]$ or $R\left[x_{1}, \ldots, x_{i-1}\right] \subset R\left[x_{1}, \ldots, x_{i-1}\right]\left[x_{i}\right]$ is minimal. As $R \subset R_{1}$ is pointwise minimal ramified, all saturated chains from $R$ to $R_{1}$ have the same length. It follows that $\ell\left[R, R_{1}\right] \leq n$. Therefore, if $n<p^{\ell_{1}}, R_{1} \subset S$ is not pointwise minimal.

We finally show that, under the conditions of the previous corollary, $R_{1} \subset S$ may be pointwise minimal.

Corollary 5.19 Let $R^{\prime}$ be a ring between $R$ and $S$ such that $R^{\prime} / M$ is a field $K$ such that $[L: K]=p^{i}, I$ be an ideal of $S$ above $M$ and contained in $N$, and $R_{1}:=R^{\prime}+I$. Then, $R_{1} \subset S$ is pointwise minimal. Moreover, if $\alpha^{\prime}:=\operatorname{dim}_{L}(I / M)$, then $\ell\left[R^{\prime}, R_{1}\right]=\alpha^{\prime} p^{i}$.

Proof It is clear that $I=N \cap R_{1}$ (only maximal ideal of $R_{1}$ above $M$ ). By Proposition 5.12, as $I$ is an ideal of $S$, it follows that $R_{1} \subset S$ is pointwise minimal. The extension $R^{\prime} \subset S$ is also pointwise minimal, so is of course $R^{\prime} \subset R_{1}$. As $R_{1}:=R^{\prime}+I, R^{\prime} \subset R_{1}$ is ramified, and by Proposition 5.8, $\ell\left[R^{\prime}, R_{1}\right]=\operatorname{dim}_{K}(I / M)=$ $\operatorname{dim}_{L}(I / M)[L: K]=\alpha^{\prime} p^{i}$. 


\section{Miscellaneous and examples}

\subsection{Examples}

Ramified pointwise minimal extensions We first give a collection of examples of (ramified) pointwise minimal extensions that are not pointwise minimal pairs.

Example 6.1 Let $k$ be a field with $\operatorname{char}(k)=2$, and $\left\{X_{i}\right\}_{i \in I}$ be a set of indeterminates. Set $S:=$ $k\left[\left\{X_{i}\right\}_{i \in I}\right] /\left(\left\{X_{i}^{2}\right\}_{i \in I}\right)$. Denote by $x_{i}$ the image of $X_{i}$ in $S$. Then, $S$ is a local ring with maximal ideal $N:=\left(\left\{x_{i}\right\}_{i \in I}\right)$ and $S=k+N$. The hypothesis on the characteristic brings $N^{[2]}=(0)$ and hence by Proposition 4.16(1), $k \subset S$ is always a pointwise minimal integral extension. It is obviously minimal if (and only if) $|I|=1$. From then on, we assume that $|I| \geq 2$ and detail additional properties.

(1) The extension $k \subset S$ is never a pointwise minimal pair: for $i \neq j, x_{i} x_{j} \neq 0$, thus $N^{2} \neq(0)$. If $|I|=2$, $S$ however admits a minimal system of two generators, showing that in the last statement of Proposition 3.9, one cannot replace pointwise minimal pair by pointwise minimal extension (Remark 3.10).

(2) It is easy to see that $k \subset S$ is finite if and only if $I$ is finite. By Proposition 3.6, it follows that $k \subset S$ has FCP (equivalently has FMC) if and only if $I$ is finite. By Proposition 3.7, $k \subset S$ has FIP and if only and if both $k$ and $I$ are finite. If $I$ is infinite, $k \subset S$ is a pointwise minimal extension failing to have FCP. If $I$ is finite but $k$ is infinite, $k \subset S$ is an FCP pointwise minimal extension failing to have FIP.

We give also a collection of examples of (ramified) pointwise minimal pairs.

Example 6.2 Let $L$ be any field and $\left\{X_{i}\right\}_{i \in I}$ be a set of indeterminates. Set $S:=L\left[\left\{X_{i}\right\}_{i \in I}\right] /\left(\left\{X_{i}^{2}, X_{i} X_{j}\right\}_{i, j \in I}\right)$ and denote by $x_{i}$ the image of $X_{i}$ in $S$. Then, $S$ is a local ring with maximal ideal $N:=\left(\left\{x_{i}\right\}_{i \in I}\right)$ and $S=L+N$. By construction, $N^{2}=(0)$ and $L \subset S$ is a pointwise minimal pair. As above, we assume that $|I| \geq 2$ (otherwise, the extension is minimal).

(1) The extension is co-pointwise minimal if and only if $|I|=2$. Indeed, it follows by Proposition 3.9 that $L \subset S$ is co-pointwise minimal if and only if $S=L[x, y]$, with $(x, y)$ a minimal system of two generators; this condition is obviously satisfied if and only if $|I|=2$.

(2) As above, the extension has FCP, if and only if $I$ is finite, and the extension has FIP and if only and if both $k$ and $I$ are finite. In case $I$ is infinite, $L \subset S$ is a pointwise minimal pair failing to have FCP. In case $|I|=2$ and $k$ is infinite, $L \subset S$ is a co-pointwise minimal extension failing to have FIP.

\section{Inert type}

Example 6.3 Let $F$ be a field of characteristic $p$ and $\left\{X_{i}\right\}_{i \in I}$ be a set of indeterminates. Let $S:=k\left(X_{i}\right)_{i \in I}$ be the field of rational functions over $k$ in these indeterminates, and $k:=F\left(X_{i}^{p}\right)_{i \in I}$. As $\operatorname{char}(k)=p$, it follows that $k \subset S$ is a height one radicial field extension, that is, $S^{[p]} \subseteq k$. In particular, $k \subset S$ is an inert pointwise minimal extension, and also a pointwise minimal pair. If $I=\{1,2, \ldots, n\}$ then

$$
k \subset k\left(X_{1}\right) \subset k\left(X_{1}, X_{2}\right) \subset \cdots \subset k\left(X_{1}, X_{2}, \ldots, X_{n}\right)=S .
$$

The length of the extension is $n$ and $[S: k]=p^{n}$.

Composite type By Proposition 4.20, it is easy to give examples of pointwise minimal extension of the composite type.

Example 6.4 Let $k$ be a field of prime characteristic $p, k \subset L$ be a field extension such that $L^{[p]} \subseteq k$, and $L \subset S$ be a pointwise ramified extension as in Example 6.2, or Example 6.1 if $p=2$. Then $k \subset S$ is a pointwise minimal extension of the composite type. In particular, $S$ is local with maximal ideal $N$. It is easy to see that in Example 6.2, $\operatorname{dim}_{L}(N)=|I|$. In case $k \subset S$ is finite, $\operatorname{dim}_{L}(N)$ can be arbitrarily chosen and $[L: k]$ be any power of $p$. Otherwise, both $\operatorname{dim}_{L}(N)$ or $[L: k]$ can be infinite.

In particular, one can start from $L:=\mathbb{F}_{2}(Y)$ (in one indeterminate) and set $k=\mathbb{F}_{2}\left(Y^{2}\right)$. Then $\operatorname{char}(k)=2$, the extension $k \subset L$ is minimal and $[L: k]=2$. One can choose $S:=L[X] /\left(X^{2}\right)$ (and denote by $x$ the class of $X)$. Then $\operatorname{dim}_{L}(S)=2$. In that case $\alpha=1, \ell_{1}=1$, and $p=2$. By Proposition 5.16, $\ell[k, S]=\alpha p^{\ell_{1}}+\ell_{1}=3$. In particular, if $N$ denotes the maximal ideal of $S$, the chain $k \subset k[x] \subset k+N \subset S$ has length 3 . On the other hand, $k \subset L \subset S$ is a saturated chain of length 2 .

We illustrate Remarks 4.6 and 4.19 with a negative example. 
Example 6.5 Let $k$ be a field of characteristic 2, with an element $a$ that is not a square. Set $S:=k[X] /\left(X^{4}+a^{2}\right)$ and denote by $x$ the class of $X$. Thus $S=k[x]$ and $x$ is of degree 4. Clearly, $S$ is local with principal maximal ideal $N$, generated by $t:=x^{2}+a$ and $N^{2}=(0)$. It follows that $k+N=k+k t$ is a $k$-vector space of dimension 2. Hence $k \subset k[x]$ is not minimal (since $k \subset k+N \subset S=k[x]$ ) and $k \subset S$ is not pointwise minimal. However $S / N \cong k[X] /\left(X^{2}+a\right)$ and $S / N$ is such that $(S / N)^{[2]} \subset k$.

As $k \subset S$ is not pointwise minimal, $k \subset S$ cannot be radicial of height one: in fact, it is obvious that $x^{2} \notin k$. Also, by Proposition 4.20, $S$ does not contain any field isomorphic to $S / N$. Therefore $S$ does not have any inert element: if $y \in S$ were inert, $k[y]$ would be isomorphic to a subfield of $S / N$, thus in fact isomorphic to $S / N$ since $[(S / N): k]=2$.

\subsection{Transfer properties with respect to Nagata rings}

Given a ring $R$, recall that the Nagata ring $R(X)$ is the localization $R(X)=T^{-1} R[X]$ of the ring of polynomials $R[X]$ with respect to the multiplicative set $T$ formed by the polynomials of content $R$ (that is, such that the ideal generated by their coefficients is $R$ ). It is known that $R(X) \subset S(X)$ is a minimal extension if and only if $R \subset S$ is minimal, of the same type [6, Theorem 3.4] (more generally, the length is preserved [12, Theorem 3.3]). Moreover, if $R \subset S$ is an integral extension, then $S(X)=T^{-1} S[X]$ [6, Lemma 3.1 (e)]. We then look at transfer properties of pointwise minimal extensions (resp. pointwise minimal pairs).

But why not consider also polynomial rings? In fact, it is easy to show that $R[X] \subset S[X]$ is never a pointwise minimal extension. First, $R[X] \subset S[X]$ is never a minimal extension. Indeed, one has $R[X] \subset$ $R+X S[X] \subset S[X]$. If $R[X] \subset S[X]$ is integrally closed, it thus follows immediately from Proposition 3.5 that $R[X] \subset S[X]$ is not pointwise minimal. On the other hand, it is classical (and easy to show) that, if $M=(R: S)$ then $M[X]=(R[X]: S[X])$. Hence the conductor $(R[X]: S[X])$ is never a maximal ideal of $R[X]$. In the integral case, it thus follows from Theorem 3.2 that $R[X] \subset S[X]$ is not pointwise minimal.

We can then turn to Nagata rings. If $R \subset S$ is integrally closed, equivalently if $R(X) \subset S(X)$ is integrally closed, $R \subset S$ is pointwise minimal (in fact, minimal), if and only if so is $R(X) \subset S(X)$ [6, Theorem 3.4]. We then suppose that $R \subset S$ is integral, equivalently that $R(X) \subset S(X)$ is integral. To examine the transfer property of pointwise minimal (integral) extensions we use Theorem 5.4. The first condition for $R \subset S$ to be a pointwise minimal extension is that there exists a maximal ideal $M$ of $R$ such that $M S=M$. We thus look at the crucial ideal, that is, the conductor of the extension. We have the following from general properties of Nagata rings.

Lemma 6.6 Let $R \subset S$ be an extension and $M:=(R: S)$ be the conductor of the extension. Then, $M(X)=$ $(R(X): S(X))$. Moreover, $M$ is maximal in $R$ if and only if $M(X)$ is maximal in $R(X)$.

In other words, there exists a maximal ideal $M$ of $R$ such that $\mathrm{MS}=\mathrm{M}$ if and only if there exists a maximal ideal of $R(X)$, necessarily equal to $M(X)$, such that $M(X) S(X)=M(X)$. We next turn to other conditions of Theorem 5.4. Unlike the conductor property, these conditions hold for polynomial rings when they hold for Nagata rings.

Lemma 6.7 Let $R \subset S$ be a ring extension.

(1) Let $I$ be an ideal of $S$. The following are equivalent:
(a) $R+I=S$,
(b) $R[X]+I[X]=S[X]$,
(c) $R(X)+I(X)=S(X)$.

(2) Let $I$ be an ideal of $S$ and $M$ an ideal of $R$. The following are equivalent:

(a) $I^{[2]} \subseteq M$ (resp. $I^{2} \subseteq M$ ),

(b) $(I[X])^{[2]} \subseteq M[X]\left(\operatorname{resp} .(I[X])^{2} \subseteq M[X]\right)$,

(c) $(I(X))^{[2]} \subseteq M(X)\left(\operatorname{resp} .(I(X))^{2} \subseteq M(X)\right)$.

(3) Assume that $M:=(R: S)$ is a maximal ideal of $R$ and that $\operatorname{char}(R / M)=p$. The following are equivalent:

(a) $S^{[p]} \subseteq R$

(b) $(S[X])^{[p]} \subseteq R[X]$,

(c) $(S(X))^{[p]} \subseteq R(X)$.

Proof (1) Assume that $R+I=S$. Let $P=\sum_{i=0}^{n} a_{i} X^{i} \in S[X]$. By hypothesis, for each $i$, one can write each $a_{i}=b_{i}+c_{i}$, with $b_{i} \in R, c_{i} \in I$. Thus $P=F+G$, with $F=\sum_{i=0}^{n} b_{i} X^{i} \in R[X]$ and $G=\sum_{i=0}^{n} c_{i} X^{i} \in I[X]$. Therefore $R[X]+I[X]=S[X]$. 
Assume that $R[X]+I[X]=S[X]$. Let $f=P / Q \in S(X)$ with $P \in S[X], Q \in R[X]$ with content $R$. Then, $f=(F+G) / Q \in S(X)$, with $F \in R[X]$ and $G \in I[X]$. That is, $f=F / Q+G / Q$, with $F / Q \in R(X)$ and $G / Q \in I(X)$. Therefore, $R(X)+I(X)=S(X)$.

Assume that $R(X)+I(X)=S(X)$. Let $x \in S$. As $S \subset S(X)$, one can write $x=(F+G) / Q$, with $F \in R[X], G \in I[X]$, and $Q \in R[X]$ with content $R$. Write $Q(X)=\sum_{j=0}^{m} b_{j} X^{j}$. Then $x b_{j} \in R+I$, for each $j$. As the content of $Q$ is $R$, there exist $\lambda_{1}, \ldots, \lambda_{m} \in R$ with $\sum_{i=0}^{m} \lambda_{j} b_{j}=1$, giving $x \in R+I$.

(2) Assume that $I^{[2]} \subseteq M$. Let $P=\sum_{i=0}^{n} a_{i} X^{i} \in I[X]$. Then, $a_{i} \in I$ for each $i=0, \ldots, n$. The coefficients of $P^{2}$ are either of the form $a_{i}^{2}$ or $2 a_{i} a_{j}$ for $i \neq j$. The hypothesis implies directly that $a_{i}^{2} \in M$, for each $i$. The relation $\left(a_{i}+a_{j}\right)^{2}=a_{i}^{2}+a_{j}^{2}+2 a_{i} a_{j}$ next implies that $2 a_{i} a_{j} \in M$. Thus $P^{2} \in M[X]$.

Assume that $(I[X])^{[2]} \subseteq M[X]$. Let $f \in I(X)$. Then $f=P / Q$ with $P \in I[X]$ and $Q \in R[X]$ with content $R$. Then, $f^{2}=P^{2} / Q^{2}$, with $P^{2} \in(I[X])^{[2]} \subseteq M[X]$ gives $f \in M(X)$.

Assume that $(I(X))^{[2]} \subseteq M(X)$. Let $x \in I$. As $I \subseteq I(X)$, it follows that $x^{2} \in M(X) \cap S=M$.

We leave to the reader the (very similar) proof of the equivalences $I^{2} \subseteq M,(I[X])^{2} \subseteq M[X],(I(X))^{2} \subseteq$ $M(X)$.

(3) Assume that $S^{[p]} \subseteq R$. As $M=(R: S)$, this is equivalent to say that $(S / M)^{[p]} \subseteq R / M$. To show that $(S[X])^{[p]} \subseteq R[X]$, we prove that $((S / M)[X])^{[p]} \subseteq(R / M)[X]$. Let $P(X)=\sum_{i=0}^{n} a_{i} X^{i}$ be a polynomial with coefficients in $S / M$. As $\operatorname{char}(R / M)=\operatorname{char}(S / M)=p$, we have $P^{p}=\sum_{i=0}^{n} a_{i}^{p} X^{p i}$. By hypothesis, $a_{i}^{p} \in(R / M)$ for each $i$. We are done.

Assume that $(S[X])^{[p]} \subseteq R[X]$. Let $f=P / Q \in S(X)$, with $P \in S[X]$ and $Q \in R[X]$ with content $R$. Then, $f=P^{p} / Q^{p}$, with $P^{p} \in R[X]$ gives $f(X) \in R(X)$.

Assume that $(S(X))^{[p]} \subseteq R(X)$. Let $x \in S$. Then, $x^{p} \in R(X) \cap S=R$.

From Lemmas 6.6 and 6.7, we will see that pointwise minimal extensions (and pairs) transfer to Nagata rings for all types but the decomposed one (unless $R \subset S$ is a minimal extension). We start with this negative answer.

Proposition 6.8 Let $R \subset S$ be an integral extension. The following are equivalent:

1. $R(X) \subset S(X)$ is a pointwise minimal decomposed extension,

2. $R(X) \subset S(X)$ is a minimal decomposed extension,

3. $R \subset S$ is a minimal decomposed extension.

Proof The last two statements are equivalent by [6, Theorem 3.4]. A minimal extension is pointwise minimal, thus it remains to show that $R(X) \subset S(X)$ is never a pointwise minimal decomposed extension, unless it is minimal. Suppose that $R(X) \subset S(X)$ is a pointwise minimal decomposed extension. Let $M(X)$ be the crucial maximal ideal of the extension. By Theorem 5.4, either $R(X) \subset S(X)$ is minimal or $R(X) / M(X) \cong \mathbb{F}_{2}$. The second case never occurs as $R(X) / M(X)$ is an infinite field.

We now prove the transfer property, in all other cases.

Proposition 6.9 $R \subset S$ is a pointwise minimal extension (resp. pointwise minimal pair) of the ramified, inert, or composite type if and only if $R(X) \subset S(X)$ is a pointwise minimal extension (resp. pointwise minimal pair) of the same type.

Proof If $R \subset S$ is a pointwise minimal (integral) extension there is a maximal ideal $M$ of $R$ such that $M S=M$ and then $M(X)$ is a maximal ideal of $R(X)$ such that $M(X) S(X)=M(X)$ (Lemma 6.6). This holds also if $R(X) \subset S(X)$ is a pointwise minimal (integral) extension. If moreover $R \subset S$ is not decomposed, there is a single maximal ideal $N$ of $S$ above $M$ and accordingly a single maximal ideal $N(X)$ of $S(X)$ above $M(X)$. This also holds if $R(X) \subset S(X)$ is not decomposed. We can thus assume there is such a maximal ideal $M$ of $R$ and such a maximal ideal $N$ of $S$. We then prove for each case (ramified, inert, composite) that the other conditions of Theorem 5.4 are satisfied for $R \subset S$ if and only if there are satisfied for $R(X) \subset S(X)$.

- Ramified type: under our assumptions, $R \subset S$ is a ramified pointwise minimal extension (resp. pair) if and only if there is an ideal $I$ of $S$ such that $S=R+I$ and $I^{[2]} \subseteq M$ (resp. $\left.I^{2} \subseteq M\right)$ (and one can take $\left.I=N\right)$. By Lemma 6.7, these conditions are satisfied if and only if $S(X)=R(X)+I(X)$ and $(I(X))^{[2]} \subseteq M(X)$ (resp. $(I(X))^{2} \subseteq M(X)$ ) (and one can replace $I(X)$ by $N(X)$ ). Thus $R \subset S$ is a ramified pointwise minimal extension (resp. pair) if and only if the same holds for $R(X) \subset S(X)$.

- Inert type: $R \subset S$ is an inert pointwise minimal extension (and a pointwise minimal pair) if and only if $S / M$ is a field and either $R / M \subset S / M$ is a separable minimal field extension or char $(R / M)=p$ and 
$S^{[p]} \subseteq R$. Now $S / M$ is a field if and only if $S(X) / M(X)$ is a field, $R / M \subset S / M$ is a separable minimal field extension if and only if $R(X) / M(X) \subset S(X) / M(X)$ is a separable minimal field extension [12, Proposition 2.4]), $\operatorname{char}(R / M)=\operatorname{char}(R(X) / M(X))$, and $S^{[p]} \subseteq R$ if and only if $S(X)^{[p]} \subseteq R(X)$ by Lemma 6.7. Thus $R \subset S$ is an inert pointwise minimal extension (and pair) if and only if $R(X) \subset S(X)$ is an inert pointwise minimal extension (and pair).

- Composite type: $R \subset S$ is a composite pointwise minimal extension if and only if $R \subset R+N \subset S$ and $S^{[p]} \subseteq R$, where $p=\operatorname{char}(R / M)$. By Lemma 6.7, the first condition is equivalent to $R(X) \subset$ $R(X)+N(X) \subset S(X)$ and the second one is equivalent to $S(X)^{[p]} \subseteq R(X)$. Thus $R \subset S$ is a composite pointwise minimal extension if and only if $R(X) \subset S(X)$ is a composite pointwise minimal extension. (Recall that a composite pointwise minimal extension is never a pointwise minimal pair).

Remark 6.10 Suppose that $R \subset S$ is a ramified pointwise minimal extension: there is an ideal $I$ of $S$ such that $S=R+I$ and $I^{[2]} \subseteq M$. By Lemma 6.7, $S[X]=R[X]+I[X]$ and $(I[X])^{[2]} \subseteq M[X]$. All conditions of Theorem 5.4 are then met to conclude that $R[X] \subset S[X]$ is a ramified pointwise minimal extension, but the condition on the conductor (Remark 5.5). Similarly in the inert and composite case, $R[X] \subset S[X]$ fails to be pointwise minimal only because the conductor of the extension is not a maximal ideal of $R[X]$.

By Proposition 3.9, co-pointwise minimal extensions are pointwise minimal pairs of length 2 . As the length of extensions transfers to Nagata rings [15, Theorem 3.3], one finally derives immediately the following from Corollary 5.9 that characterizes co-pointwise minimal extensions.

Corollary 6.11 $R(X) \subset S(X)$ is a co-pointwise minimal extension if and only if $R \subset S$ is a co-pointwise minimal extension of the ramified or inert type. In that case $R(X) \subset S(X)$ is of the same type as $R \subset S$.

\subsection{Quadratic extensions}

An extension $R \subseteq S$ is said to be quadratic if $R[t]=R+R t$, for each $t \in S$. We say that a nontrivial element $t \in S$ is quadratic if $R[t]=R+R t$. Thus, a quadratic extension is an extension such that every element is either quadratic or trivial. It is clear that a quadratic extension is an integral extension.

We wish to compare quadratic and (integral) pointwise minimal extensions.

If $R$ and $S$ share an ideal $I$, we can use the fact that the properties of $R \subset S$ are reflected in $R / I \subset S / I$ (Proposition 2.8). In particular:

Lemma 6.12 Let $R \subset S$ be a pair of rings sharing an ideal I. Then $R \subset S$ is quadratic if and only if $R / I \subset S / I$ is quadratic.

To examine if an integral pointwise minimal extension with crucial ideal $M$ is quadratic, we can thus consider $R / M \subset S / M$ and reduce to the case of an extension over a field. For minimal extensions we immediately have the following:

Lemma 6.13 A minimal field extension $k \subset S$ is quadratic if and only if it is either decomposed, ramified, or inert and $k \subset S$ is a quadratic field extension, that is, $[S: k]=2$.

We derive the following.

Theorem 6.14 (A) Let $R \subset S$ be a quadratic extension. Then $R \subset S$ is pointwise minimal, if and only if there is a maximal ideal $M$ of $R$ such that $M S=M$.

(B) Let $R \subset S$ be an integral pointwise minimal extension with crucial maximal ideal $M$. Then $R \subset S$ is quadratic if and only if one of the following mutually exclusive conditions is satisfied.

(1) $R \subset S$ is decomposed,

(2) $R \subset S$ is ramified,

(3) $R \subset S$ is inert and either $[S / M: k]=2$, or $\operatorname{char}(k)=2$,

(4) $R \subset S$ is composite and $\operatorname{char}(k)=2$.

Proof (A) Assume that $R \subset S$ is a quadratic extension, in particular, it is an integral extension. If $R \subset S$ is a pointwise minimal extension, there is a maximal ideal $M$ of $R$ such that $M S=M$. Conversely, assume there is a maximal ideal $M$ of $R$ such that $M S=M$. Consider the extension $k:=R / M \subset S / M$. Let $t$ be a nontrivial element of $S / M$. Since $t$ is quadratic, $\operatorname{dim}_{k}(k[t])=2$, hence $t$ is minimal. Thus, $R \subset S$ is a pointwise minimal extension. 
(B) Assume that $R \subset S$ is an integral pointwise minimal extension with crucial maximal ideal $M$. The extension $k:=R / M \subset S / M$ is pointwise minimal of the same type. If $R \subset S$ is either decomposed or ramified, it is quadratic since every nontrivial element of $S / M$ is quadratic by the previous lemma. If $k \subset S / M$ is an inert pointwise minimal extension, then either $k \subset S / M$ is a minimal separable field extension or $\operatorname{char}(k)=p$ and, for each minimal element $t \in S / M,[k[t]: k]=p$. Thus $k \subset S / M$ is quadratic if and only if either $[S / M: k]=2$ or $\operatorname{char}(k)=2$. In the composite case, $\operatorname{char}(k)=p$, and every element is minimal, either ramified or inert. The ramified elements are quadratic, the inert ones are quadratic if and only if $\operatorname{char}(k)=2$.

We end with an example of a quadratic extension that is far from being pointwise minimal.

Example 6.15 The extension $\mathbb{Z} \subset \mathbb{Z}[\sqrt{d}]$ ( $d$ not a square in $\mathbb{Z}$ ) is quadratic: for each $t \in \mathbb{Z}[\sqrt{d}]$, one can write $t=a+b \sqrt{d}$, with $a, b \in \mathbb{Z}$, and $t^{2}=\left(d b^{2}-a^{2}\right)+(2 a) t$. Yet, $\mathbb{Z} \subset \mathbb{Z}[\sqrt{d}]$ is not pointwise minimal. In fact, there is no minimal element. If $t=a+b \sqrt{d}$ is a nontrivial element, then $b \neq 0$, and the conductor of the extension $\mathbb{Z} \subset \mathbb{Z}[t]$ is (0) since the quotient field of $\mathbb{Z}$ is $\mathbb{Q}$ and the quotient field of $\mathbb{Z}[t]$ is $\mathbb{Q}(\sqrt{d})$. As the extension $\mathbb{Z} \subset \mathbb{Z}[t]$ is integral, it is not a minimal extension.

Open Access This article is distributed under the terms of the Creative Commons Attribution 4.0 International License (http:// creativecommons.org/licenses/by/4.0/), which permits unrestricted use, distribution, and reproduction in any medium, provided you give appropriate credit to the original author(s) and the source, provide a link to the Creative Commons license, and indicate if changes were made.

\section{References}

1. Bourbaki, N.: Algèbre, Chs 4-7. Masson, Paris (1981)

2. Cahen, P.-J.: Couples d'anneaux partageant un idéal. Archiv der Math. 51, 505-514 (1988)

3. Cahen, P.-J.; Dobbs, D.E.; Lucas, T.G.: Characterizing minimal ring extensions. Rocky Mt. J. Math. 41(4), 1081-1125 (2011)

4. Cahen, P.-J.; Dobbs, D.E.; Lucas, T.G.: Valuative domains. J. Algebra Appl. 9, 43-72 (2010)

5. Dobbs, D.E.; Picavet, G.; Picavet-L'Hermitte, M.: Characterizing the ring extensions that satisfy FIP or FCP. J. Algebra 371, 391-429 (2012)

6. Dobbs, D.E.; Picavet, G.; Picavet-L'Hermitte, M.: Transfer results for the FIP and FCP properties of ring extensions. Commun. Algebra 43, 1279-1316 (2015)

7. Dobbs, D.E.; Shapiro, J.: Patching together a minimal overring. Houston J. Math. 36, 985-995 (2010)

8. Ferrand, D.; Olivier, J.-P.: Homomorphismes minimaux d'anneaux. J. Algebra 16, 461-471 (1970)

9. Grothendieck, A.; Dieudonné, J.A.: Eléments de Géométrie Algébrique I. Springer, Berlin (1971)

10. Picavet, G.; Picavet-L'Hermitte, M.: Morphismes t-clos. Commun. Algebra 21, 179-219 (1993)

11. Picavet, G.; Picavet-L'Hermitte, M.: T-closedness. In: Chapman, S.T., Glaz, S. (eds.) Non-Noetherian Commutative Ring Theory, Mathematics and Its Applications, vol. 520, pp. 369-386. Springer, New York (2000)

12. Picavet, G.; Picavet-L'Hermitte, M.: Some more combinatorics results on Nagata extensions. Palest. J. Math. 5, 49-62 (2016)

13. Picavet-L'Hermitte, M.: Minimal order morphisms. J. Number Theory 45, 1-27 (1993)

14. Swan, R.G.: On seminormality. J. Algebra 67, 210-229 (1980)

15. Traverso, C.: Seminormality and Picard group. Ann. Scuola Norm. Sup. Pisa 24, 585-595 (1970)

Publisher's Note Springer Nature remains neutral with regard to jurisdictional claims in published maps and institutional affiliations. 